\title{
Nématodes parasites \\ de Caméléons malgaches
}

\section{Deuxième note}

Par Alain-G. CHABAUD et Edouard-R. BRYGOO

Nous avons, au cours d'un premier travail (1960), signalé l'existence de neuf espèces de Nématodes chez les Caméléons malgaches; un Rhabdias a été décrit en 1961. Les très nombreuses autopsies pratiquées récemment portent le nombre des espèces à 20. Elles se répartissent de la façon suivante :

\section{RHABDITIDA.}

\section{RHABDITINA.}

\section{Rhabditoidea.}

\section{Rhabdiasidae.}

Rhabdias gemellipara Chab., Bryg. et Petter 1961.

C. parsonii Cuv., Perinet.

C. brevicornis Günth., Perinet, Ambavaniasy, Ifanadia, Ampamaherana, Fiherenana.

C. lateralis Gray, expérimental.

STRONGYLINA.

\section{Trichostrongyloidea.}

\section{Trichostrongylidae.}

\section{Molineinae.}

Oswaldocruzia legendrei $\mathrm{n}$. sp.

C. brevicornis Günth., Mandraka.

C. lateralis Gray, Mandraka.

Brookesia superciliaris (Kuhl), Ambavaniasy. 


\section{ASCARIDIDA.}

\section{Cosmocercoidea.}

\section{Cosmocercidae.}

\section{Cosmocercinae.}

Raillietnema travassosi n. sp.

C. nasutus Dum. et Bibr., Ambavaniasy.

Raillietnema vicarians $\mathrm{n}$. $\mathrm{sp}$.

C. gallus Günth., Ambavaniasy.

Raillietnema deblocki $\mathrm{n}$. sp.

C. parsonii Cuv., Ambavaniasy.

Raillietnema dupuisi $\mathrm{n}$. sp.

C. parsonii Cuv., Vavatenina.

Raillietnema oligogenos $\mathrm{n}$. sp.

C. minor Günth., Belo-sur-Mer.

\section{Maxvachoniinae.}

Maxvachonia dimorpha Chab. et Bry. 1960.

C. pardalis Cuv., Nossi-Bé.

\section{Kathlaniidae.}

Falcaustra golvani Chabaud et Brygoo 1957.

Amphibiens, nombreuses localités.

C. brevicornis Günth., Ambavaniasy.

\section{Oxyuroidea.}

\section{Oxyuridae.}

\section{Pharyngodoninae.}

Pharyngodon dimorpha $\mathrm{n}$. sp.

Lézards, Zampongotra.

C. pardalis Cuv., Fort Dauphin.

Thelandros meridionalis $\mathrm{n}$. $\mathrm{sp}$.

Lézards, région Tuléar, Zampongotra.

Serpents, Etrobeka.

C. verrucosus Cuv., Etrobeka, Manombo.

C. lateralis Gray., Amboasary.

Thelandros sp. Chabaud et Brygoo 1960.

C. pardalis Cuv., Nossi-Bé.

\section{Ascaridoidea.}

\section{Ascarididae.}

Hexametra angusticaecoides Chabaud et Brygoo 1960.

Caméléons variés, nombreuses localités. 


\section{Heterakoidea.}

\section{Heterakidae.}

Spinicaudinae.

Spinicauda (Spinicauda) inglisi Chabaud et Brygoo 1960.

Caméléons variés, nombreuses localités.

Spinicauda (Moaciria) freitasi.

Caméléons variés, nombreuses localités.

Strongyluris radaodyi Chabaud et Brygoo 1960.

Caméléons variés, nombreuses localités.

\section{SPIRURIDA.}

\section{Physalopteroidea.}

\section{Physalopteridae.}

Physalopteroides chamaeleonis Chabaud et Brygoo 1960.

Caméléons variés, nombreuses localités.

Abbreviata madagascariensis Chabaud et Brygoo 1960.

Caméléons variés, nombreuses localités.

\section{Filarioidea.}

\section{Onchocercidae.}

\section{Dirofilariinae.}

Foleyella furcata (Linstow 1895).

Caméléons variés, nombreuses localités.

Foleyella brevicauda n. sp.

C. brevicornis Günth., Perinet, Ambavaniasy.

C. parsonii Cuv., Ambavaniasy.

Nous allons décrire ici les espèces non encore étudiées dans les notes précédentes (Chabaud et Brygoo, 1960 ; et Chabaud, Brygoo et Petter, 1961) et ajouterons quelques remarques concernant certaines des espèces déjà connues.

\section{Rhabdias gemellipara Chabaud, Brygoo et Petter 1961}

L'espèce a été décrite récemment chez $C$. parsonii Cuvier à Périnet, et l'infestation expérimentale a été possible chez $C$. lateralis Gray.

La longueur de la femelle type et des autres spécimens du même lot était d'environ $8,4 \mathrm{~mm}$.

Depuis cette première récolte, un Rhabdias de très grande taille a été trouvé chez $C$. brevicornis Günth. en différentes localités: Ampamaherana (13 mars 1962), Ifanadiana (22 février 1962), Ambavaniasy (10 février 1962), Périnet (février 1962). Les dimensions sont habituellement très différentes de celles 
que nous avions observées chez les parasites de $C$. parsonii. Un grand spécimen mesure : Longueur : $30 \mathrm{~mm}$. Largeur : 1,52 mm. Capsule buccale haute de $70 \mu$ et large de $40 \mu$. Esophage : $1.650 \mu$. Anneau nerveux à $400 \mu$ de l'apex. Vulve à $18,45 \mathrm{~mm}$. de la pointe caudale. Queue : $400 \mu$.

Nous pensons cependant que l'espèce peut être assimilée à gemellipara car l'œsophage, qui a un aspect assez caractéristique, semble identique. Nous redonnons une vue apicale de la tête car les papilles céphaliques sont plus nettement visibles (fig. $10 \mathrm{~b}$ ).

\section{OSW ALDOCRUZIA LEGENDREI n.SP.}

De nombreux spécimens mâles et femelles ont été récoltés dans l'estomac et l'intestin antérieur d'un Chamaeleo brevicornis Günth. de la Mandraka en février 1961 (hôte type).

Quatre caméléons de la même région ont été examinés : un brevicornis et trois lateralis. Deux étaient parasités: un brevicornis et un lateralis (mars 61). Par contre, à Ambavaniasy, sur quatre Brookesia superciliaris capturés, deux étaient porteurs de $O$. legendrei (novembre et décembre 61) tandis que 41 caméléons appartenant à huit espèces différentes n'hébergeaient pas ce parasite.

\section{Description}

Corps grêle recouvert d'une cuticule ornée de 28 à 30 fortes stries longitudinales et de très fines stries transversales. La cuticule céphalique, largement dilatée, forme une ampoule subsphérique. De très petites ailes latérales, à peine plus hautes que les stries longitudinales, sont discernables dans la région œsophagienne.

Bouche triangulaire, entourée d'un cadre très faiblement chitinoïde. Deux amphides et quatre papilles submédianes, grandes et saillantes. Le lobe œsophagien dorsal apparaît, en vue apicale, plus foncé que les lobes latéraux ventraux, mais il n'y a pas de dent chitinoïde dorsale. L'œsophage, assez court, comprend un tiers antérieur cylindrique, un tiers moyen légèrement rétréci en isthme, et un tiers postérieur dilaté en massue. L'anneau nerveux est un peu en avant du milieu de l'œsophage, le pore excréteur à peu près au niveau de son extrémité postérieure, et les diérides un peu en arrière.

\section{Mâle.}

Corps long de 4,3 mm., large de $95 \mu$. Esophage long de $375 \mu$ avec anneau nerveux, pore excréteur et diérides respectivement à $170 \mu, 325 \mu$ et $375 \mu$ de l'apex. Les spicules longs de $170 \mu$ sont formés d'un manche proximal et de trois lames distales. La lame dorsale se termine en pointe aiguë, les deux lames latérales sont symétriques et se terminent par une pointe en forme de crochet. 


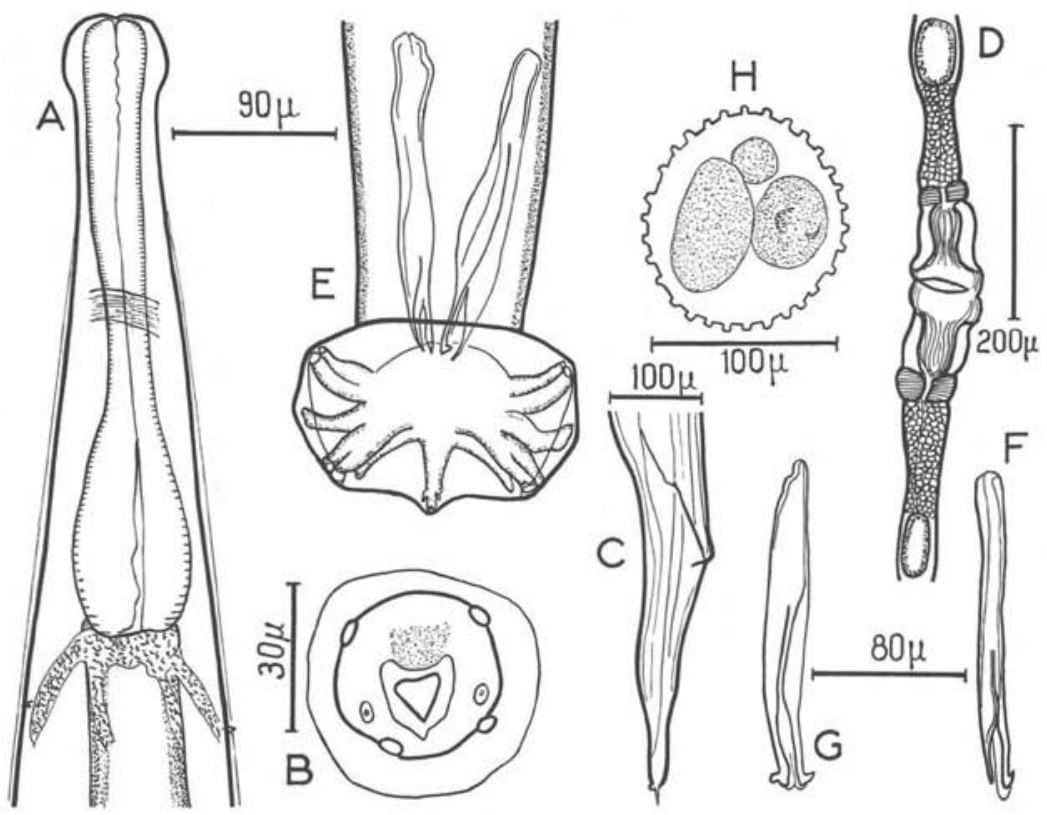

FIg. 1. - Oswaldocruzia legendrei. A) Femelle, extrémité antérieure, vue ventrale. - B) Femelle, tête, vue apicale. - C) Femelle, queue, vue latérale. - D) Dissection de l'ovéjecteur. - E) Mâle, extrémité postérieure, vue ventrale. - F) Dissection d'un spicule, vue latérale. - G) Id., vue ventrale. - H) Femelle, coupe du corps montrant les stries longitudinales.

La bourse caudale est représentée sur la figure 2. Le cône génital est de forme arrondie simple. Une petite languette charnue, insérée au niveau de la base, pend librement devant sa face ventrale.

\section{Femelle.}

Corps long de $6,5 \mathrm{~mm}$., large de $140 \mu$. Esophage long de $360 \mu$ avec anneau nerveux, pore excréteur, et diérides respectivement à $165 \mu, 340 \mu$ et $390 \mu$ de l'apex.

Queue longue de $260 \mu$, portant à sa pointe une épine terminale bien marquée et deux tubercules très courts, si bien qu'il semble y avoir là une transition entre les structures de type Strongylacantha et les structures de type Molineus.

Vulve à $4,1 \mathrm{~mm}$. de l'extrémité antérieure. Ovéjecteur composé des trois parties habituelles, figuré en $1 \mathrm{D}$. Eufs très segmentés, longs de $68 \mu$ et larges de $38 \mu$. 


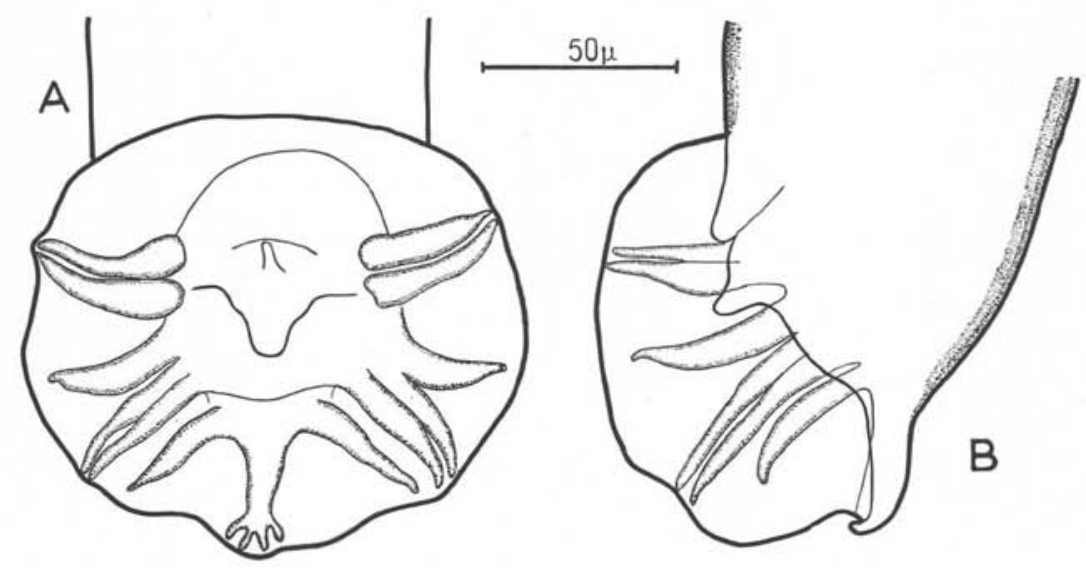

FIG. 2. - Oswaldocruzia legendrei. Bourse caudale du mâle A) Vue ventrale. - B) Vue latérale

\section{Discussion}

Bien que cette forme ait tous les caractères du genre Oswaldocruzia, elle présente plusieurs particularités qui font qu'elle est facile à différencier des nombreuses espèces déjà décrites.

Ce sont :

a) le parasitisme chez un Caméléon ;

b) la forme de l'œsophage rétréci en isthme dans sa partie moyenne;

c) la queue de la femelle portant deux petits tubercules en plus de la pointe terminale ;

d) la constitution des spicules: les spicules de la plupart des espèces comprennent à leur terminaison une pointe dorsale, une pointe latéro-ventrale obtuse en forme de pied, une pointe latéro-ventrale aiguë et deux pointes accessoires fines annexées aux lames ventrales. Ici, ces pointes accessoires sont absentes et les deux pointes ventrales sont symétriques et en forme de crochet.

Nous pensons donc que l'espèce est nouvelle, et proposons pour elle le nom d'Oswaldocruzia legendrei n. sp., en hommage amical au Professeur R. Legendre, à qui nous devons la découverte de cette espèce.

\section{Genre RAILLIETNEMA}

Le genre Raillietnema fut créé par Travassos en 1927 pour des Aplectana ayant un petit nombre d'œufs dans les utérus. Ce caractère, d'importance apparemment minime, s'accompagne cependant d'une réduction des ovaires qui, comme l'indique Travassos, semble faire de ce genre un lien entre Cosmocercidae et Atractidae. 
Bien que rejeté dans certains ouvrages modernes, ce genre a été admis par beaucoup des auteurs qui se sont intéressés au groupe (et, en particulier, Walton, 1940 ; Ballesteros, 1945, et Fotedar, 1960).

Nous disposons ici de cinq espèces, et quatre sont parfaitement homogènes et conformes au générotype. Par contre, une $5^{\circ}$, bien qu'extrêmement proche des précédentes, a des œufs un peu plus nombreux et ses ovaires s'allongent et deviennent presque aussi longs que ceux des Aplectana. Il est regrettable de ne pas conserver le genre Raillietnema, qui forme un groupe homogène intéressant puisqu'il semble localisé à l'hémisphère austral, mais nous devons admettre que les caractères morphologiques qui le séparent d'Aplectana sont discutables, et nous ne le retenons ici qu'à titre provisoire.

Il est curieux de constater que les espèces « communes » de Caméléons, dont un grand nombre d'exemplaires a été examiné, n'ont jamais fourni de Raillietnema.

Ce sont quatre espèces de Caméléons rarement autopsiés, qui ont été trouvés positifs et ont procuré cinq espèces de Raillietnema.

Parmi celles-ci, une a été récoltée à trois reprises (toujours chez le même hôte et dans la même localité), mais les quatre autres n'ont été trouvées qu'une seule fois.

Les cinq espèces sont excessivement proches les unes des autres, et proches également d'un parasite de Chamaeleo melleri (Gray) du Nyassaland, Raillietnema chamaeleo Fitzsimmons 1961. Cette dernière espèce ayant été étudiée de façon très détaillée, les descriptions peuvent être abrégées.

\section{Le matériel comprend:}

1) De nombreux mâles, femelles, et deux femelles juvéniles, de trois Chamaeleo nasutus Dum. et Bib., provenant d'Ambavaniasy (avril, juin et juillet 1961). Nous proposons de les nommer Raillietnema travassosi n. sp., en l'honneur du grand helminthologiste Lauro Travassos.

2) Trois mâles et cinq femelles d'un Chamaeleo gallus Günther, provenant d'Ambavaniasy (avril 1961). Nous proposons de les nommer Raillietnema vicarians $\mathrm{n}$. $\mathrm{sp}$.

3) De très nombreux spécimens, mâles et femelles, d'un Chamaeleo parsonii Cuvier, provenant d'Ambavaniasy (décembre 1961). Nous proposons de les nommer Raillietnema deblocki n. sp., en cordial hommage à Stéphane Deblock, Professeur agrégé de Parasitologie à Lille.

4) De très nombreux spécimens, mâles, femelles et larves, d'un Chamaeleo parsonii Cuvier, de Vavatenina (mai 1959). Nous proposons de les nommer Raillietnema dupuisi n. sp., en cordial hommage à Claude Dupuis, Sous-Directeur du Laboratoire des Vers.

5) D'assez nombreux spécimens, mâles, femelles et larves, d'un Chamaeleo minor Günther, provenant de Belo-sur-Mer (août 1961). Nous proposons de les nommer Raillietnema oligogenos $\mathrm{n}$. sp. 


\section{Caractères communs}

Quelle que soit l'espèce, ce sont des Nématodes de très petite taille, couverts d'une cuticule fine, à stries transversales à peine visibles à l'immersion. Deux ailes latérales très étroites débutent à peu près au niveau de l'anneau nerveux et restent perceptibles jusque dans la région postérieure.

L'extrémité céphalique (non étudiée en vue apicale chez vicarians) est assez plate. La bouche est petite et triangulaire. Les amphides et les quatre papilles submédianes sont bien saillantes et assez proches de la bouche. Il semble y avoir chez travassosi quatre papilles médio-médianes un peu antérieures aux submédianes, mais l'existence de ces papilles ne peut être affirmée, et elle ne peut évidemment pas être donnée comme caractère différentiel. Chaque lèvre est doublée en profondeur par une forte dent pharyngée, presque aussi haute que la lèvre elle-même.

L'œsophage est constitué par un pharynx d'un diamètre peu différent du corpus, mais de structure différente. De la même façon, la limite corpus-isthme peut être décelée par la couleur plus que par un changement de diamètre. Le bulbe lui-même est subsphérique et distinctement valvulé. Le pore excréteur n'a aucun renforcement chitinoïde. La vulve est très postérieure. L'ovéjecteur est très court. Il se dirige vers l'avant et comprend un tube musculeux, puis une trompe impaire à parois minces. La disposition des tubes génitaux est caractéristique et parfaitement constante: le tube génital antérieur est dirigé directement vers l'avant; le postérieur se dirige vers l'arrière, puis se courbe à mi-trajet et prend un trajet récurrent vers l'extrémité antérieure. L'ovaire postérieur se trouve donc finalement à peu près au niveau de l'ovéjecteur. Sauf chez $R$. deblocki, les ovaires sont remarquablement courts. Les utérus ne contiennent qu'un très petit nombre d'œufs qui éclosent généralement dans la partie terminale des utérus.

L'extrémité postérieure des mâles est dépourvue d'ailes caudales. Les papilles comprennent un groupe presque constant de trois paires et d'une impaire formant un cercle juste en avant du cloaque. Les précloacales antérieures sont plus variables par leur nombre et par leur disposition. Les postcloacales varient également un peu d'une espèce à l'autre, mais certaines sont très petites et difficiles à déceler, et l'on ne peut considérer leur présence ou leur absence comme un caractère différentiel. Les spicules et le gubernaculum sont de forme très simple, mais leurs dimensions varient nettement d'une espèce à l'autre.

\section{Caractères différentiels et discussion}

Deux Oxysomatium sont connus chez des Caméléons en Afrique : $O$. perezi (Gendre 1911) et $O$. chamaeleonis Baylis 1929.

La structure génitale femelle n'a pas été précisée dans les descriptions originales et l'extrémité postérieure du mâle s'éloigne peu de ce que nous obser- 


\begin{tabular}{|c|c|c|c|c|c|c|}
\hline 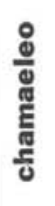 & 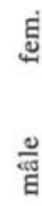 & 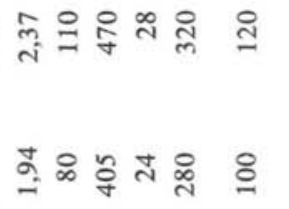 & 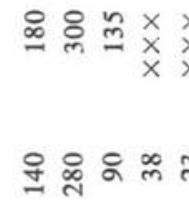 & $\begin{array}{l}\vec{n} \\
- \\
x \\
x \\
x\end{array}$ & 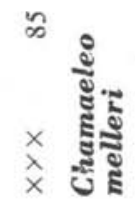 & \\
\hline 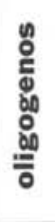 & लै & 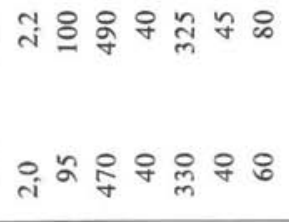 & 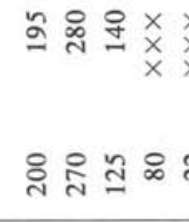 & $\begin{array}{l}\stackrel{f}{ \pm} \\
\dot{x} \\
\dot{x} \\
x\end{array}$ & 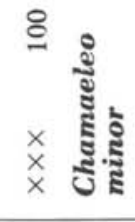 & 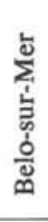 \\
\hline $\begin{array}{l}\bar{n} \\
\text { 를 }\end{array}$ & 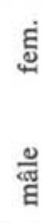 & 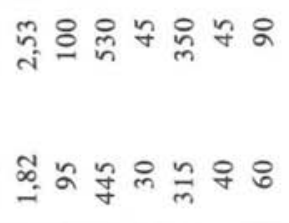 & 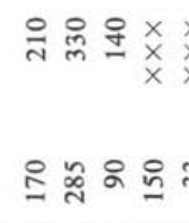 & $\begin{array}{l}\hat{b} \\
- \\
x \\
x \\
x\end{array}$ & 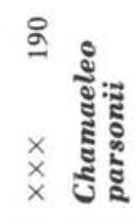 & .ّ \\
\hline$\frac{\bar{x}}{\frac{\pi}{2}}$ & ฮึ & 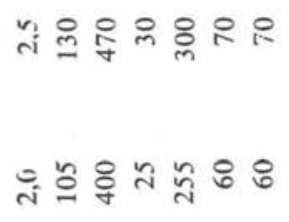 & 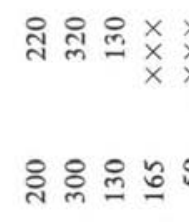 & $\begin{array}{l}\stackrel{x}{x} \\
\dot{x}\end{array}$ & 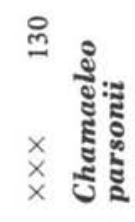 & 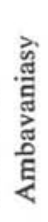 \\
\hline 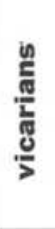 & ๕్ & 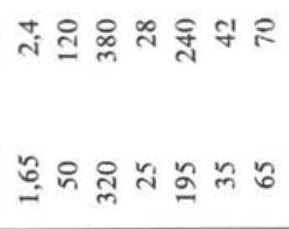 & 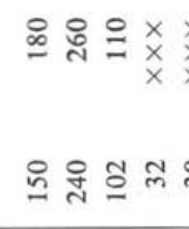 & $\begin{array}{l}\sim \\
- \\
x \\
x \\
x\end{array}$ & : & 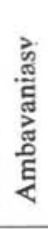 \\
\hline 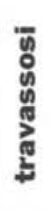 & 丞 & 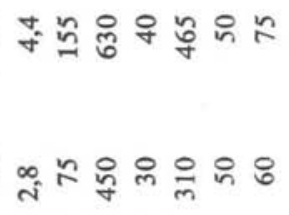 & 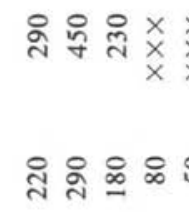 & $\begin{array}{l}\stackrel{x}{x} \\
\times\end{array}$ & 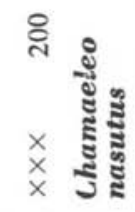 & 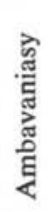 \\
\hline \multicolumn{7}{|c|}{ 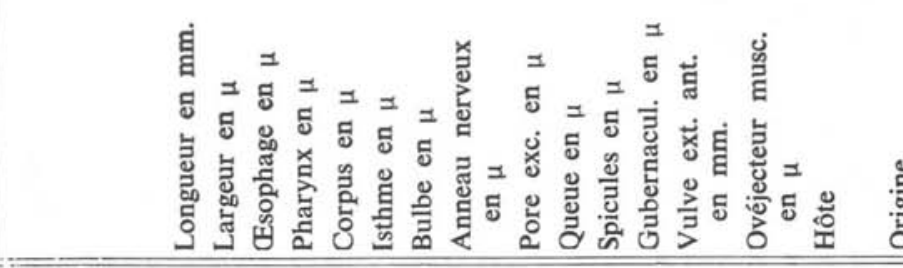 } \\
\hline
\end{tabular}


vons sur notre matériel. Cependant, il s'agit d'espèces de grande taille qui appartiennent donc vraisemblablement au genre Oxysomatium sensu stricto.

A notre connaissance, le genre Raillietnema ne compte actuellement que cinq ou six espèces: une espèce type sud-américaine, une ou deux espèces australiennes et trois espèces du Sud-Est de l'Afrique. Ce sont :

- R. simples (Travassos 1925), parasite de Hyla au Brésil.

- R. kartanum Johnston et Mawson 1941, et $R$. flindersi (Johnston et Mawson 1941), tous deux parasites de Hyla en Australie. (Cette dernière espèce a été placée dans le genre Aplectana, mais la femelle est inconnue et les dimensions du mâle rendent possible l'appartenance au genre Raillietnema. Si c'est bien le cas, la diagnose avec les autres espèces du genre ne pose pas

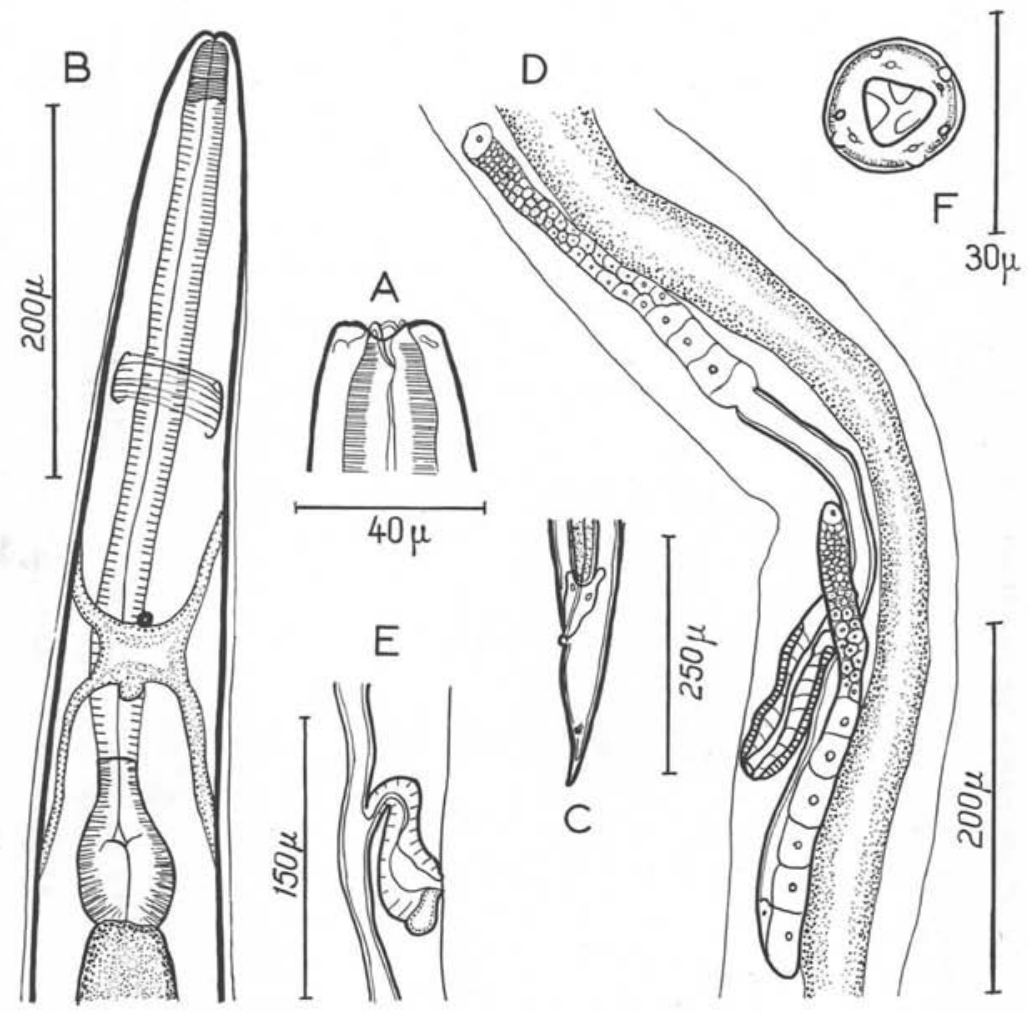

FIG. 3. - Raillietnema travassosi. A) Extrémité antérieure, mâle, vue ventro-latérale. - B) Région œsophagienne, femelle juvénile, vue ventrale. C) Extrémité postérieure, femelle juvénile, vue latérale. - D) Appareil génital, femelle juvénile. - E) Ovéjecteur, seconde femelle juvénile. - F) Tête, mâle, vue apicale, technique Anderson. 


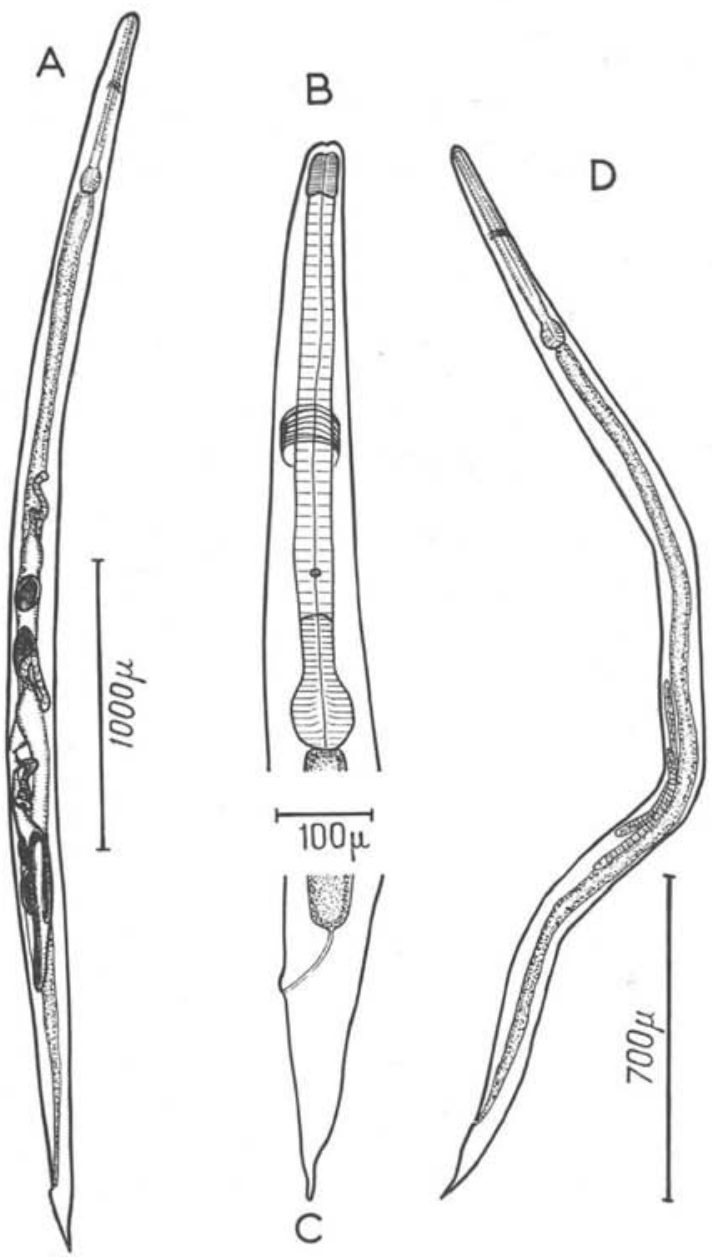

FIG. 4. - Raillietnema travassosi. A) Femelle mûre. B) Id., région œsophagienne, vue ventrale. - C) $I d$., queue, vue latérale. - D) Femelle juvénile.

de difficultés, car le gubernaculum est nettement plus grand et plus gros que les spicules).

- R. loveridgei (Sandground 1928) et $R$. multipapillata Walton 1940, tous deux parasites de Coeciliens (respectivement Bdellophis et Scolecomorphus), au Tanganyika, et enfin $R$. chamaeleo Fitzsimmons 1961, parasite de Chamaeleo melleri (Gray), au Nyassaland.

Les espèces parasites d'Amphibiens ont des spicules relativement longs Ann. de Parasitologie, T XXXVII, N ${ }^{\circ} 4 .-1962$. 
(sauf kartanum), et, d'après les descriptions de Walton (1940) et de Johnston et Mawson (1941), elles sont pourvues de petites ailes caudales (sauf flindersi) et d'un sinus excréteur chitinoïde. Elles peuvent donc être facilement séparées des espèces que nous étudions et c'est finalement $R$. chamaeleo qui apparaît comme étant le plus proche de notre matériel.

\section{I) R. TRAVASSOSI n.Sp.}

L'espèce est relativement de grande taille puisque les mâles atteignent $2,8 \mathrm{~mm}$. et les femelles $4,4 \mathrm{~mm}$.

L'extrémité postérieure du mâle est un peu enflée dans la région cloacale. La queue est relativement longue avec une pointe très fine. Les papilles cloa-

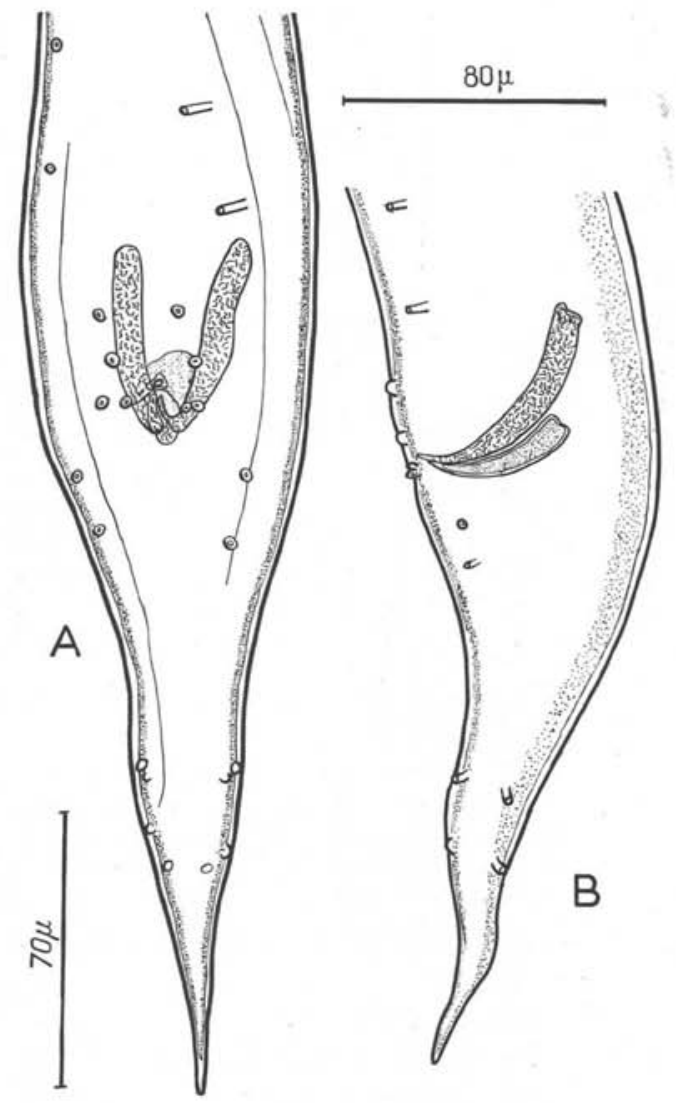

FIG. 5. - Raillietnema travassosi, mâle. Extrémité postérieure. A) Vue ventrale. - B) Vue latérale (un seul spicule a été représenté). 
cales les plus antérieures et les plus postérieures rampent un peu à la surface du corps et sont donc faiblement pédonculées, bien que la cuticule soit très mince.

Il y a 21 papilles et deux phasmides : six paires sont étagées assez régulièrement sur une hauteur de $300 \mu$ en avant du niveau de la tête des spicules; une papille impaire et quatre paires sont groupées dans la région précloacale, les deux plus postérieures étant situées sur une même ligne transversale. Parmi les postcloacales, deux paires sont situées un peu en arrière du cloaque et deux paires à peu près au même niveau, à $90 \mu$ en arrière du cloaque ; la dernière et les phasmides sont placées à $20 \mu$ plus postérieurement. Les spicules ont la
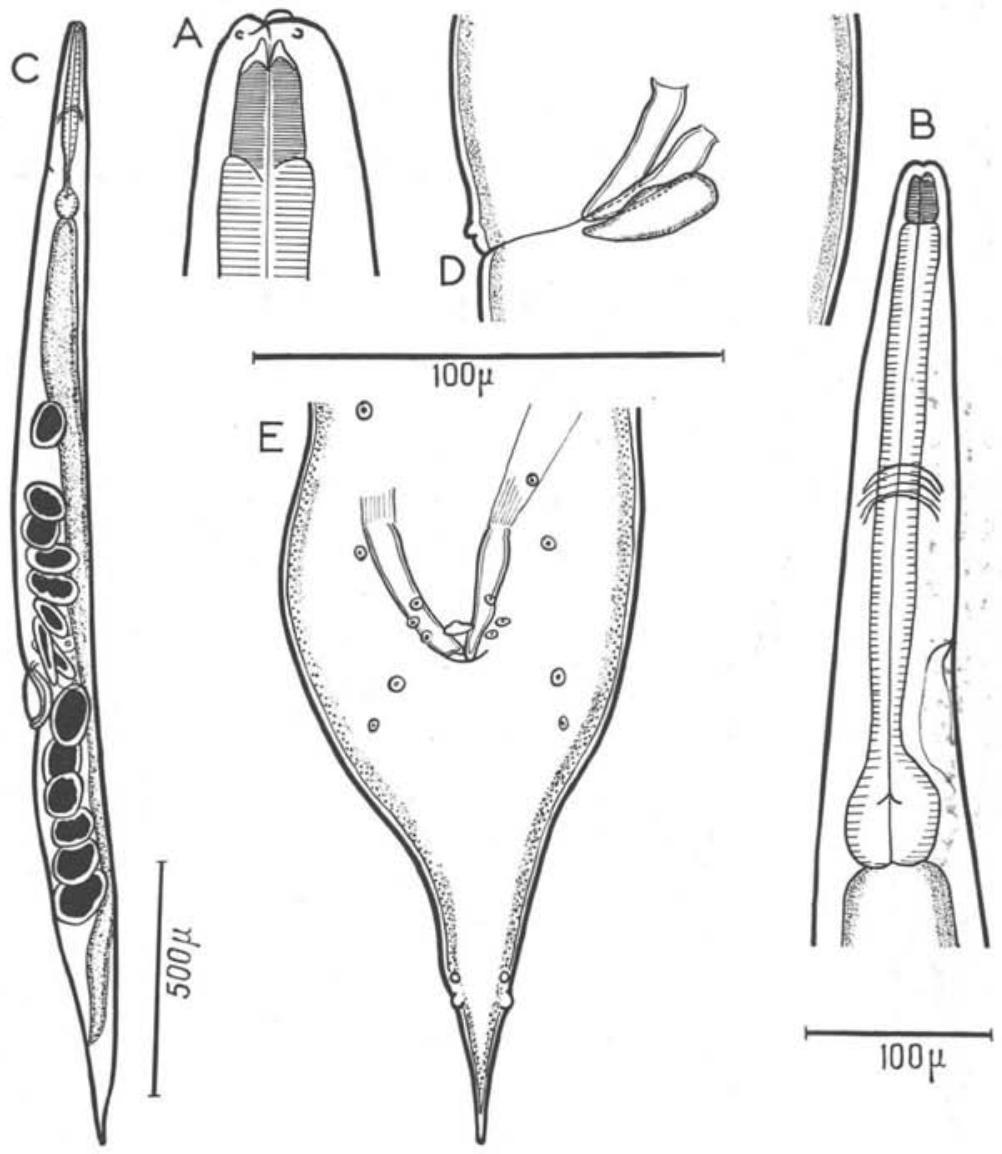

FIG. 6. - Raillietnema vicarians. A) Extrémité céphalique, femelle, vue latérale droite. - B) Esophage, femelle, vue latérale. - C) Corps entier, femelle. D) Spicules et gubernaculum, vue latérale. - E) Extrémité postérieure, mâle, vue ventrale. 
même longueur que ceux d'oligogenos $(80 \mu)$, mais ils sont plus trapus. La vulve n'est pas saillante. Les œufs ou larves sont peu abondants. On en compte deux à trois dans chaque utérus.

La diagnose la plus difficile est celle qui se pose avec vicarians, car les deux formes sont les seules qui aient un gubernaculum presque aussi long que les spicules et elles proviennent toutes les deux de la même localité. $R$. travassosi est beaucoup plus grand que vicarians; l'œsophage est relativement plus long (travassosi : $480 \mu$ chez une femelle de $2,6 \mathrm{~mm}$. ; vicarians : $380 \mu$ chez une

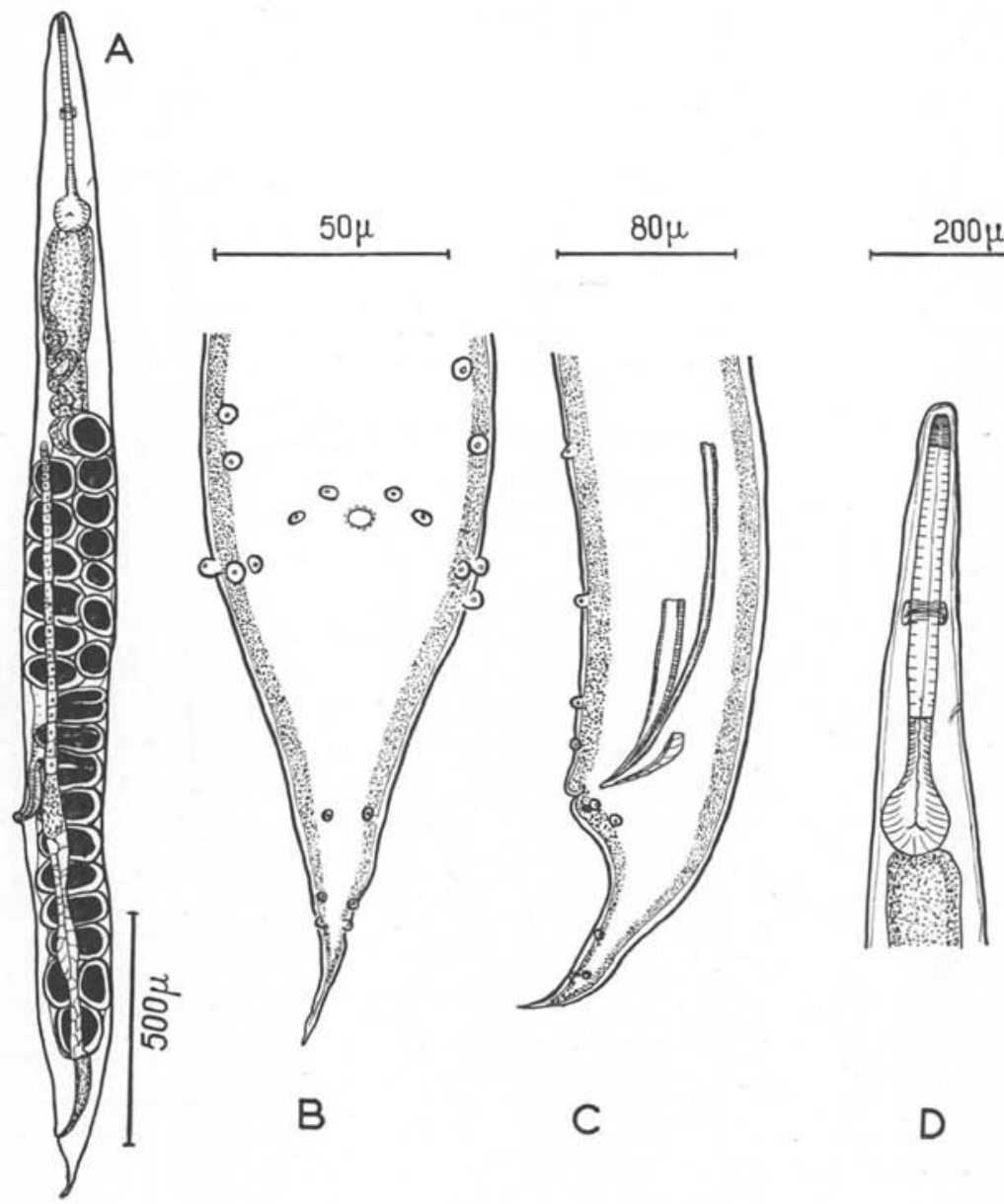

FIG. 7. - Raillietnema deblocki. A) Femelle, vue latérale. - B) Extrémité postérieure, mâle, vue ventrale. - C) Id., vue latérale d'un spécimen ayant le spicule droit atrophié. - D) Esophage, femelle, vue latérale. 
femelle de 2,4 mm.). Le nombre et la disposition des papilles cloacales semblent bien différents. Le nombre d'œufs ou de larves est nettement plus faible (4 à 6, au lieu de 10 à 16). Il semble donc impossible d'assimiler les deux formes.

\section{II) R. VICARIANS n.sp.}

Le matériel dont nous disposons est peu abondant et en moins bon état que pour les autres espèces. L'extrémité céphalique n'a pas été étudiée en détail. La vulve n'a pas de lèvres saillantes. Elle est située sur le bord postérieur d'un petit relief ventral déterminé par l'ovéjecteur. Chaque utérus contient environ six gros œufs, le dernier pouvant être remplacé par une larve éclose.

Chez le mâle, les papilles précloacales comprennent sept paires formant deux longues lignes longitudinales sur les axes latéro-ventraux, puis trois paires et une paire juste en avant du cloaque.

Les papilles postcloacales comprennent deux paires un peu en arrière du cloaque, et deux paires près de la pointe caudale. Les spicules sont égaux, très courts, peu chitinoïdes et de forme trapue. Ils sont soutenus par des ligaments très opaques qui les prolongent en arrière, et il est nécessaire d'éclaircir la préparation pour localiser leur extrémité proximale. Ils sont longs de $32 \mu$. Le gubernaculum, également très peu chitinoïde, a une forme assez globuleuse. Il est presque aussi long que les spicules $(30 \mu)$.

\section{III) R. DEBLOCKI n.sp.}

$R$. deblocki se distingue facilement des autres espèces par le nombre des œufs intra-utérins (8 à 13 dans chaque utérus) et par la longueur des ovaires et des oviductes. La disposition génitale caractéristique des Raillietnema est cependant conservée. L'ovaire et l'oviducte antérieurs sont étroitement pelotonnés en avant de l'utérus antérieur, alors qu'au contraire l'ovaire postérieur, qui naît presque au même niveau, suit un trajet parfaitement rectiligne vers l'extrémité postérieure. La vulve est assez saillante.

Le mâle possède un groupe de quatre paires de papilles précloacales latérales ; un groupe péricloacal, sur lequel deux paires seulement ont été décelées ; un groupe postcloacal avec une paire plus dorsale et deux paires latérales, et un groupe caudal également formé de trois paires dont une plus dorsale. Le gubernaculum est long de $50 \mu$ et les spicules de $165 \mu$. Normalement, ils sont égaux, mais de nombreux spécimens présentent des malformations de l'un des spicules. Le spicule atrophié a souvent un aspect « chiffonné » qui montre bien qu'il est anormal, mais, dans certains cas, il est simplement beaucoup plus court que l'autre.

Si l'on ne tient pas compte du nombre des œufs, l'espèce ressemble à dupuisi par la taille des spicules, mais l'œsophage est plus court et la disposition des papilles cloacales un peu différente. 
En dehors de travassosi dont la diagnose a été discutée plus haut, l'espèce la plus proche est $R$. chamaeleo, car les autres espèces ont des spicules plus longs et plus fins et des utérus contenant un plus petit nombre d'œufs. $R$. chamaeleo a des dimensions très comparables. On peut noter que notre espèce a un œsophage un peu plus court, une disposition des papilles cloacales et en particulier des postcloacales différente, et un gubernaculum presque aussi long que les spicules et d'aspect plus globuleux. Nous pensons donc que l'espèce est distincte.

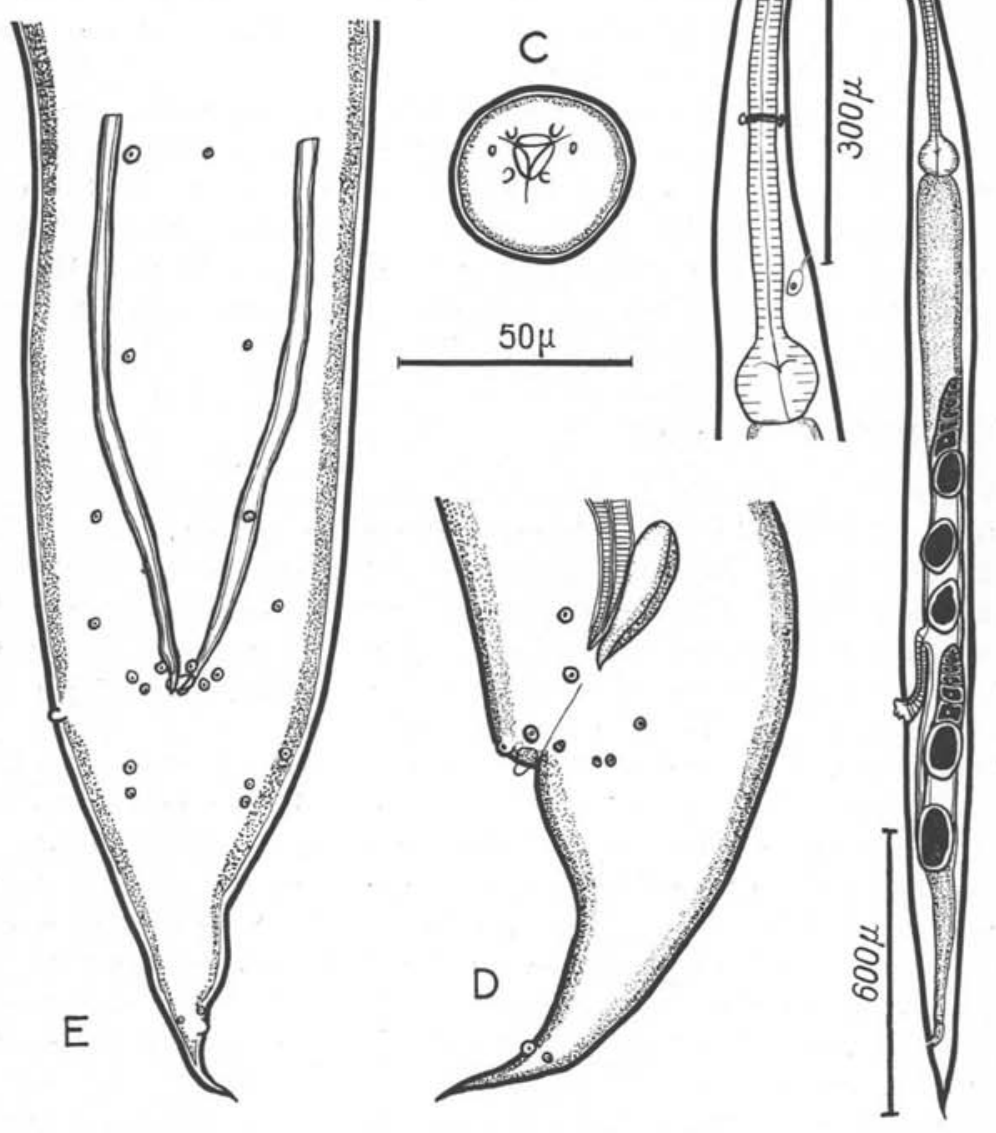

Frg. 8. - Raillietnema dupuisi. A) Femelle, vue latérale. - B) Esophage, femelle, vue latérale. - C) Tête, femelle, vue apicale. - D) Extrémité postérieure, mâle, vue latérale. - E) Id., vue ventrale. 


\section{IV) R. DUPUISI n.sp.}

L'espèce est proche de vicarians.

Sur le mâle, les différences les plus appréciables sont: $a$ ) des papilles précloacales antérieures un peu moins nombreuses (5 paires), moins espacées longitudinalement et plus proches de l'axe médio-ventral ; $b$ ) une paire très latérale, située juste en arrière du cloaque, qui existe peut-être, mais n'a pu être

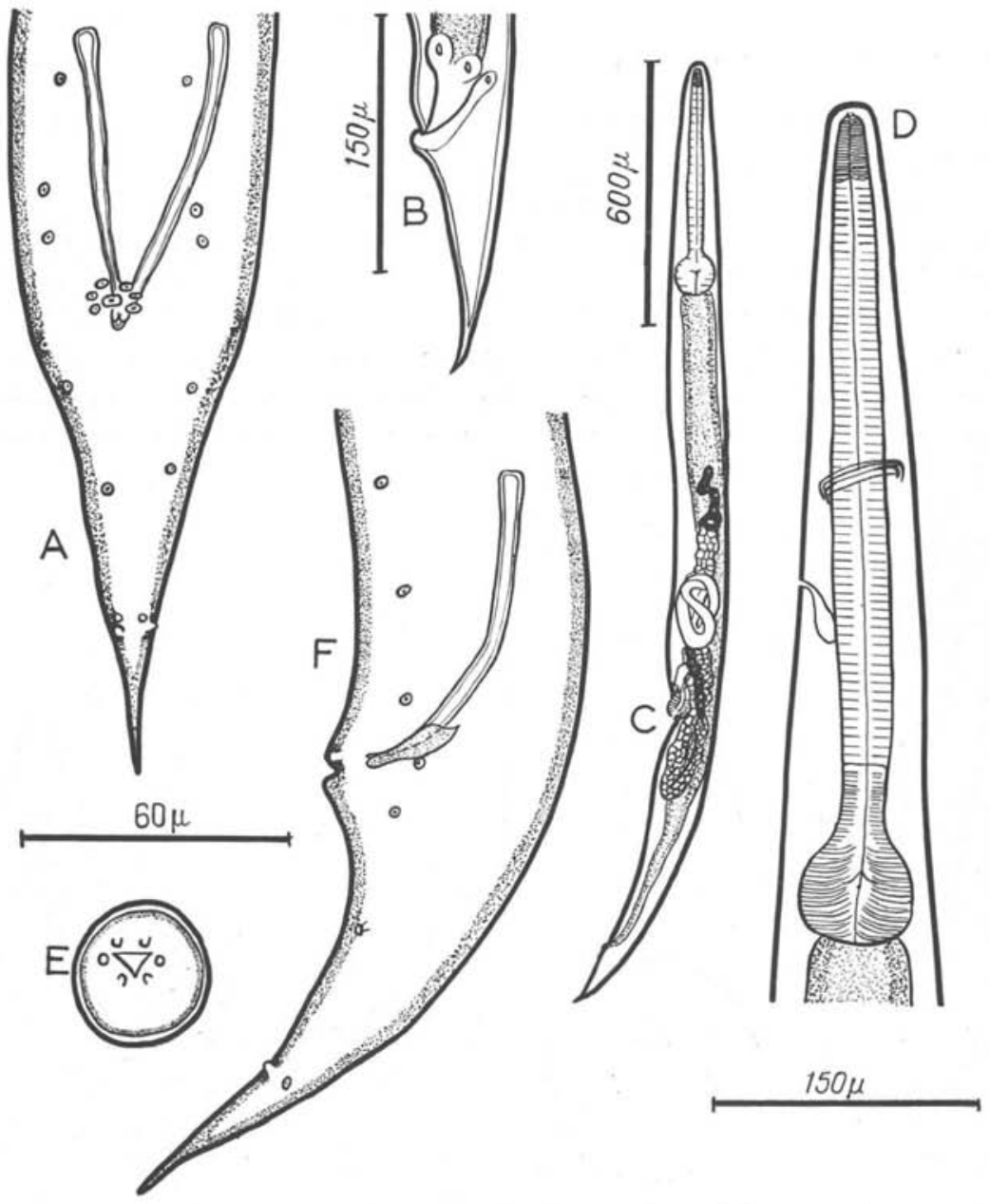

FIG. 9. - Raillietnema oligogenos. A) Extrémité postérieure, mâle, vue ventrale. - B) Queue, femelle, vue latérale. - C) Femelle, vue latérale. D) Esophage, mâle, vue latérale. - E) Tête, femelle, vue apicale. F) Extrémité postérieure, mâle, vue latérale. 
repérée sur vicarians; $c)$ des spicules beaucoup plus longs $(140 \mu$ sur le mâle type, $160 \mu$ sur d'autres spécimens); le gubernaculum est long de $33 \mu$.

Sur la femelle, la vulve a une lèvre postérieure un peu saillante et généralement marquée par une brève collerette cuticulaire festonnée. Chaque utérus ne contient que deux ou trois œufs, mais il y a presque toujours, en plus, une ou deux larves apparemment mûres.

Le nombre d'œufs intra-utérins, comparable à celui de travassosi, est donc intermédiaire entre celui de vicarians et celui d'oligogenos.

\section{V) R. OLIGOGENOS n.sp.}

Contrairement aux espèces précédentes, la femelle n'est pas nettement plus grande que le mâle.

La vulve a ses deux lèvres un peu saillantes. Le nombre des œufs ou larves présents dans les utérus est excessivement faible. Dans la grande majorité des cas, il y a seulement un œuf ou une larve dans chaque utérus, et même, sur de nombreux spécimens, l'un des deux utérus est vide.

La queue du mâle est relativement fine. Les papilles précloacales comprenrent six paires formant deux lignes longitudinales sur les axes latéro-ventraux, puis trois paires et une impaire juste en avant du cloaque. Les cinq paires
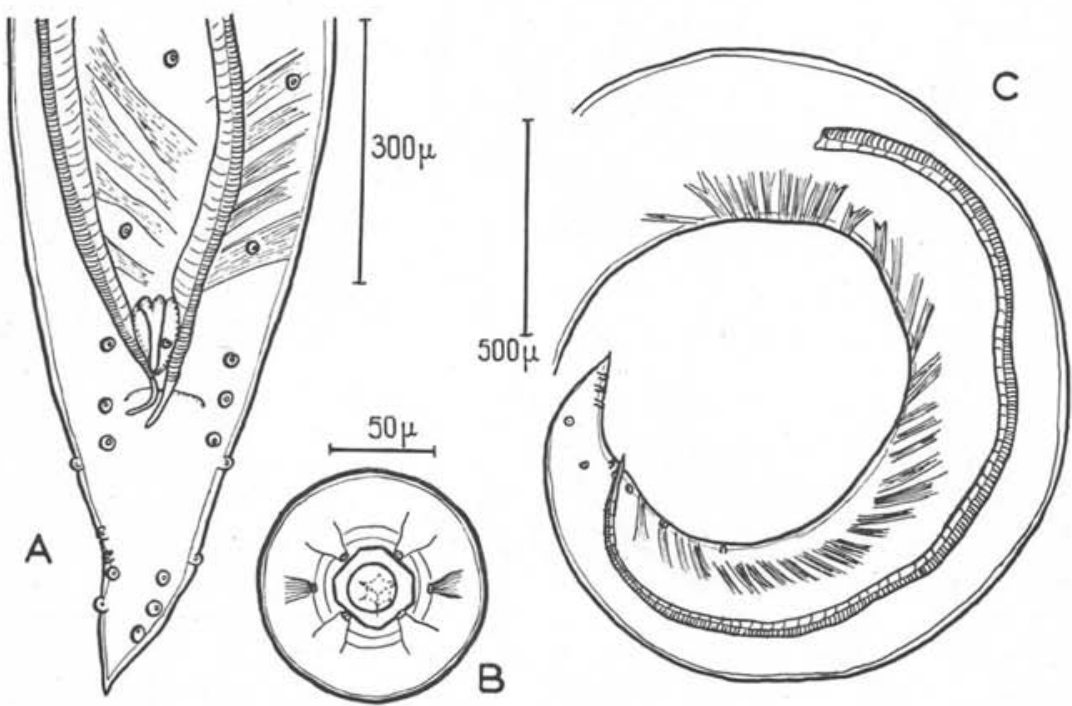

FIG. 10. - Falcaustra golvani chez Chamaeleo brevicornis d'Ambavaniasy.

Extrémité postérieure, A. vue ventrale, C. vue latérale. - B) Rhabdias gemellipara. Femelle géante chez Chamaeleo brevicornis d'Ampamaherana. Tête, vue apicale. 
post-anales sont plus régulièrement espacées que celles de dupuisi. Les spicules sont de taille moyenne, et le gubernaculum plus petit et plus grêle que précédemment.

L'espèce, bien que proche de dupuisi, est donc facile à différencier par la longueur plus réduite des spicules et par le nombre excessivement faible d'œufs intra-utérins.

\section{FALCAUSTRA GOLVANI Chabaud et Brygoo 1957}

L'espèce a été décrite chez différents Amphibiens de la région de Tananarive et a été retrouvée fréquemment en des localités variées.

Un mâle et deux femelles ont été récoltés dans (?) les poumons de Chamaeleo brevicornis Günth. à Ambavaniasy, le 10 février 1962.

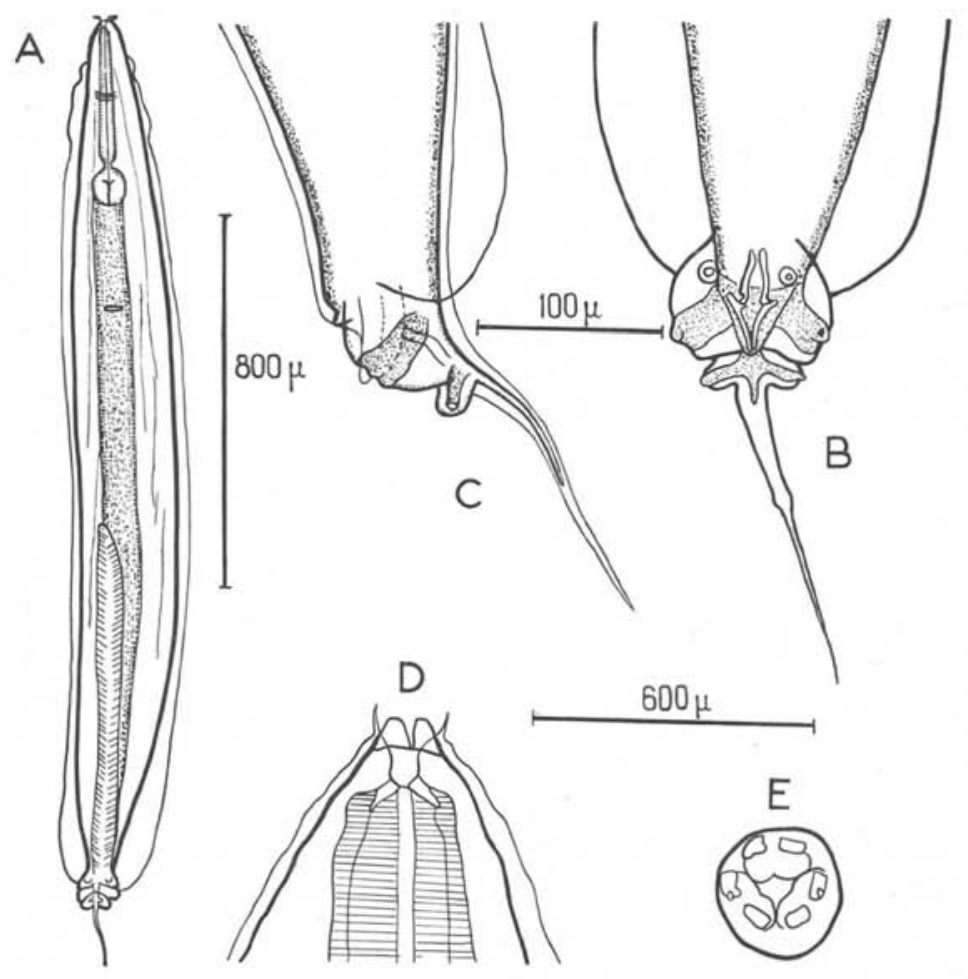

FIG. 11. - Pharyngodon dimorpha, mâle. A) Corps entier, vue ventrale. - B) Extrémité postérieure, vue ventrale. - C) Id., vue latérale. - D) Extrémité antérieure, vue dorsale. - E) Tête, vue apicale. 
Le mâle est de grande taille: $14,2 \mathrm{~mm}$., et surtout ses spicules sont très longs : 2,4 mm., au lieu de 1,2-1,3 mm. sur le spécimen type. Nous n'avons cependant relevé aucune autre différence importante avec le matériel type et pensons qu'il s'agit d'un parasite d'Amphibien égaré chez un Caméléon.

\section{PHARYNGODON DIMORPHA n.sp.}

De nombreux mâles, femelles et larves ont été récoltés dans le rectum d'un Chamaeleo pardalis Cuvier 1829, capturé à Fort-Dauphin (mai 1959). 17 autres $C$. pardalis et 4 lateralis Gray de la même région, examinés à la même époque, étaient négatifs. Par contre, l'espèce a été retrouvée chez Hoplurus cyclurus Merr. de Zampongotra. Il s'agit donc peut-être d'un parasite de Lézards égaré chez un Caméléon.

Mâle : La longueur du corps est très variable. Des spécimens longs de $1,15 \mathrm{~mm}$. sont déjà adultes et pourvus d'ailes caudales, mais la taille habituelle est d'environ $2 \mathrm{~mm}$. Le spécimen type, long de 2,05 mm., a une largeur maximum de $190 \mu$. La cuticule présente de fortes stries transversales; elle se soulève en deux puissantes ailes latérales qui naissent à $80 \mu$ de l'apex et se terminent par une forte dilatation brusquement tronquée en avant du cloaque. Tête petite, formée par trois lèvres bilobées; la base de chacune est prolongée en arrière par une paire de petites languettes transparentes, longues de $8 \mu$ et larges de $6 \mu$, dirigées vers l'avant, ou perpendiculairement à l'axe du corps. Les amphides sont situées en arrière du niveau d'insertion de ces languettes. Il semble $\mathrm{y}$ avoir un cycle interne de six papilles près du bord libre des lèvres, mais les papilles du cycle externe n'ont pas été décelées. La cavité buccale, haute de $7 \mu$, est de forme triangulaire et a des parois assez épaisses. L'œsophage est long de $350 \mu$. Le pharynx n'est pas nettement distinct du corpus. L'isthme est très court (environ $15 \mu)$. Le bulbe subsphérique $(70 \times 75 \mu)$ est pourvu de fortes valves chitinoïdes. L'anneau nerveux est situé à $125 \mu$ de l'apex et le pore excréteur à $620 \mu$. Ce dernier est renforcé par un fort anneau chitinoïde ovalaire, à grand axe transversal, mesurant $30 \mu \times 15 \mu$. L'extrémité postérieure porte de fortes ailes caudales, qui naissent ventralement aux ailes latérales, en avant des papilles préanales, et constituent une véritable petite bourse entourant dorsalement et latéralement le cône génital, et soutenues par les très gros pédoncules des papilles adanales. Les papilles postanales soulèvent également des ailes caudales, un peu plus dorsales et postérieures que les précédentes. La pointe caudale mesure $180 \mu$ de la base des papilles postanales à son extrémité. Les paires de papilles pré- et postanales ont un pédoncule épais, mais pas nettement ramifié. La paire adanale au contraire a un pédoncule très puissant, qui se termine distalement par trois ou quatre bourgeons charnus, dont un seul a une terminaison sensorielle nette. Le cône génital, fortement saillant en avant, est couvert d'une cuticule finement 


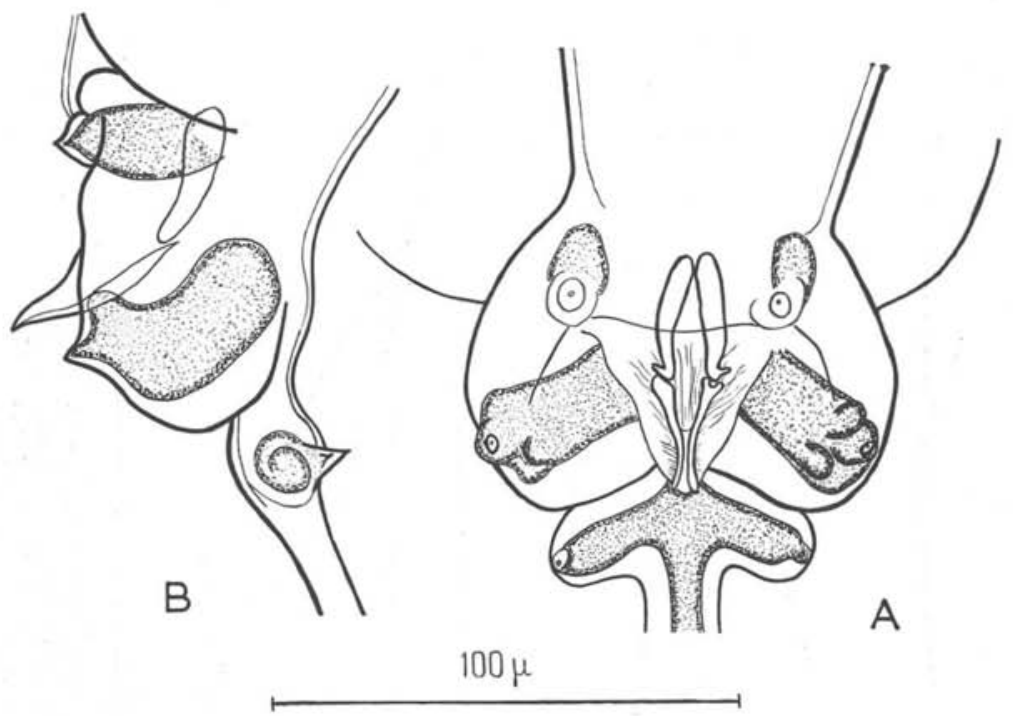

FIG. 12. - Pharyngodon dimorpha, mâle

A) Région cloacale, vue ventrale. - B) Id., vue latérale

sculptée. Il est soutenu par une pièce chitinoïde, de forme complexe, longue de $60 \mu$, qui semble articulée à sa partie moyenne.

Femelle : Une grosse femelle pleine d'œufs est longue de $4,3 \mathrm{~mm}$. et large de $350 \mu$. Il n'y a pas d'ailes latérales. La tête est constituée par trois lèvres larges de $20 \mu$, limitant une bouche triangulaire. Chaque lèvre porte une paire de mamelons saillants en avant, sur lesquels paraissent s'insérer les quatre papilles du cycle externe et, semble-t-il, les six papilles du cycle interne. Les amphides au contraire sont en arrière des mamelons latéraux. Les petites ailes transparentes qui ornent les lèvres du mâle sont absentes. L'œsophage est long de $590 \mu$. Le bulbe mesure $130 \times 130 \mu$. L'anneau nerveux, le pore excréteur et la vulve sont respectivement à 135,580 et $650 \mu$ de l'apex. Les œufs, un peu aplatis sur une face, mesurent $100 \times 41 \mu$. La queue est longue de $630 \mu$. Elle comporte une portion antérieure ayant à peu près le même diamètre que le corps, puis une pointe fine longue de $480 \mu$. Celle-ci est armée d'un petit nombre d'épines (de 4 à 7), disposées irrégulièrement.

Les dimensions d'une jeune femelle, non ovifère, sont : longueur : $2,55 \mathrm{~mm}$., largeur : $270 \mu$. Esophage long de $520 \mu$ avec bulbe de $115 \times 115 \mu$. L'anneau nerveux, le pore excréteur et la vulve sont à 110,515 et $560 \mu$ de l'apex. La queue est longue de $530 \mu$ et la pointe caudale de $430 \mu$.

L'anatomie génitale a été étudiée principalement sur des femelles de taille moyenne $(3,75 \mathrm{~mm}$.), ayant un nombre d'œufs encore modéré. L'ovéjecteur 

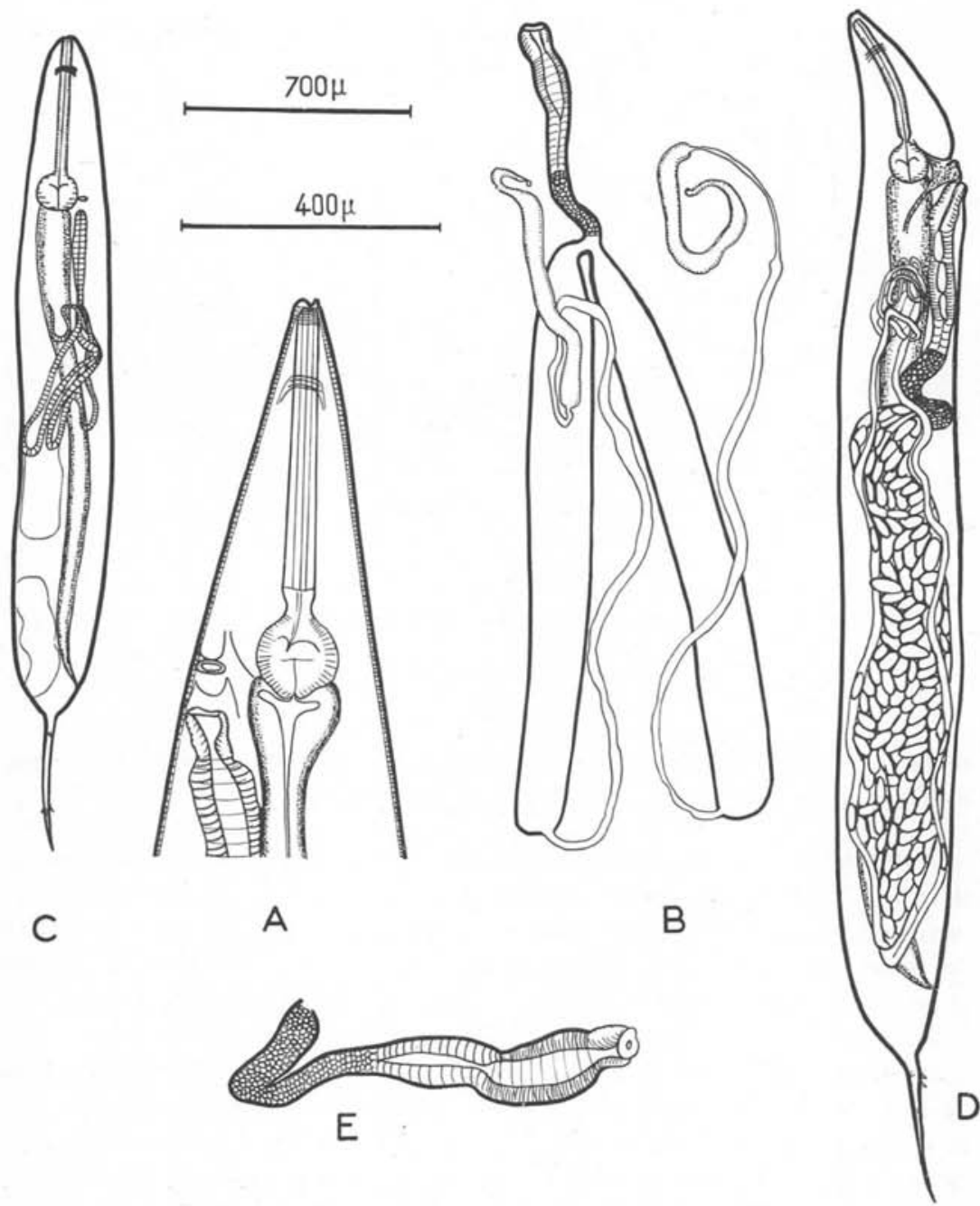

FIG. 13. - Pharyngodon dimorpha, femelle. A) Femelle de 4,3 mm. Région œsophagienne, vue sublatérale. - B) Schéma de la dissection de l'appareil génital d'une femelle de $3,75 \mathrm{~mm}$. - C) Femelle de 2,55 mm., corps entier. - D) Femelle de $3,75 \mathrm{~mm}$., corps entier. - E) Détail de l'ovéjecteur.

dirigé vers l'arrière comprend un cylindre très musculeux, long de $450 \mu$, subdivisé lui-même en une portion antérieure plus large et une portion postérieure plus étroite, puis une trompe impaire longue de $300 \mu$ qui se divise en deux utérus à parois minces. Chacun de ceux-ci comprend deux portions bien dis- 


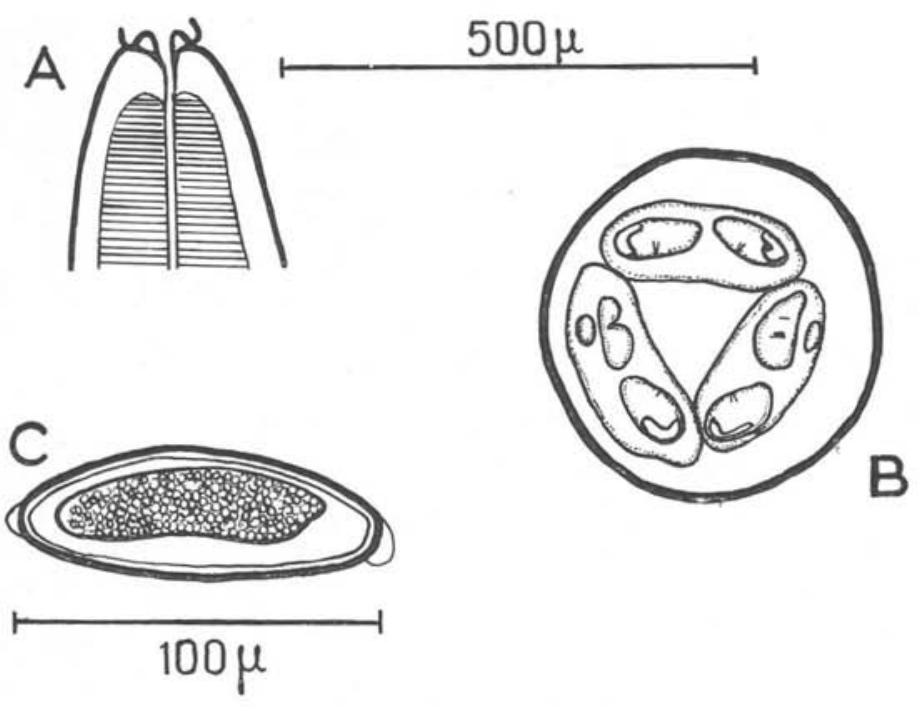

FIG. 14. - Pharyngodon dimorpha. A) Tête, larve de $710 \mu$, vue ventrale. - B) Tête, femelle de 4,0 mm., vue apicale. - C) (Euf.

tinctes: une branche descendante très dilatée, formant un vaste sac plein d'œufs, et une branche récurrente, très mince, ne contenant des œufs que sur une simple ou sur une double file. Les deux parties de l'utérus ont une longueur comparable $(1,8 \mathrm{~mm}$.) et leur union se fait à la partie la plus postérieure de leur trajet, c'est-à-dire au niveau de l'anus. Les oviductes longs de $500 \mu$ et les ovaires longs de $900 \mu$ sont enroulés au niveau de l'ovéjecteur.

\section{Discussion}

L'espèce est intéressante par son dimorphisme sexuel, car l'extrémité céphalique de la femelle semble à première vue plus simple et plus primitive que celle du mâle. Mais, dans les cas de dimorphisme connus chez les Nématodes parasites, ce sont toujours les mâles qui sont les plus primitifs. L'extrémité céphalique de la larve, pourvue de petites ailes transparentes, comme chez le mâle, montre que notre espèce n'échappe pas à la règle. La structure céphalique femelle semble donc constituer un nouvel exemple d'évolution régressive (cf. Chabaud, 1959). On peut noter également que la larve, comme le mâle et contrairement à la femelle, est pourvue de grosses ailes latérales.

A condition d'y ajouter les espèces décrites récemment par Gupta (1959), le tableau dichotomique du genre Pharyngodon, que nous avons proposé avec Y. Golvan en 1957, est utilisable, et permet de placer l'espèce malgache parmi les formes les plus primitives, presque toutes décrites d'Australie. L'es- 
pèce se rapproche de $P$. kartana Johnston et Mawson 1941, car la femelle porte des épines caudales, et de $P$. tiliquae Baylis 1930, car l'épine caudale du mâle est deux fois plus longue que la bourse. Elle est cependant facile à reconnaître, non seulement par la structure céphalique du mâle, mais encore par la pièce chitinoïde qui supporte le cône génital. Il existe chez certaines espèces, et en particulier chez $P$. tiliquae, * a supporting structure 》 dans le cône génital, mais cette formation est moins complexe et moins fortement chitinoïde que celle qui est décrite plus haut. Nous pensons donc que l'espèce malgache est nouvelle et proposons pour elle le nom de Pharyngodon dimorpha n. sp.

\section{THELANDROS MERIDIONALIS n.Sp.}

Hôtes: $1^{\circ}$ Hoplurus sp., Route Tuléar P.K. 556, 1 mâle, 4 femelles (hôte type) ; $2^{\circ}$ Hoplurus cyclurus Merr., Zampongotra, 1 mâle, 1 femelle (associé à Pharyngodon dimorpha) ; $3^{\circ}$ Acrantophis dumerilii (Ian), Etrobeka, 2 femelles; $4^{\circ}$ C. verrucosus Cuv., Manambo, Etrobeka, femelles; $5^{\circ}$ C. lateralis Gray, Nord d'Amboasary, 2 femelles mûres.

Ici encore, l'espèce pourrait être un parasite de Lézards égaré chez des Caméléons, car ceux-ci n'ont qu'une infestation faible.

\section{Description}

Mâle : Corps long de 2,9 mm., large de $300 \mu$, recouvert d'une cuticule épaisse à stries transversales espacées et profondes. Ailes latérales fortes naissant en arrière de l'œsophage et se terminant à $500 \mu$ en avant de la pointe caudale. Bouche avec trois lèvres très petites. Il semble $\mathrm{y}$ avoir deux amphides et quatre petites papilles submédianes.

Esophage total long de $430 \mu$ avec un corpus, un isthme et un bulbe larges respectivement de $32 \mu, 28 \mu$ et $110 \mu$. Anneau nerveux et pore excréteur à $125 \mu$ et $940 \mu$ de l'apex.

Spicule un peu concave ventralement, long de $80 \mu$.

La distance entre le cloaque et la dernière paire de papilles est d'environ $50 \mu$, mais la pointe caudale est de longueur variable, si bien que la longueur totale de la queue est de $128 \mu$ sur le mâle type et seulement de $80 \mu$ sur le second mâle disponible (Hoplurus cyclurus Merr. de Zampongotra). Huit papilles cloacales, dont la disposition est indiquée sur la figure 15. La petite paire postcloacale est constituée par des éléments coalescents sur la ligne médio-ventrale.

En plus des papilles, il y a une ornementation chitinoïde formant approximativement un cercle péricloacal. Elle est constituée par des éléments un peu irréguliers, se soulevant en petites languettes latérales.

Femelle : Corps de longueur variable ( 5 à $8 \mathrm{~mm}$.). Pas d'ailes latérales sur les gros spécimens. 


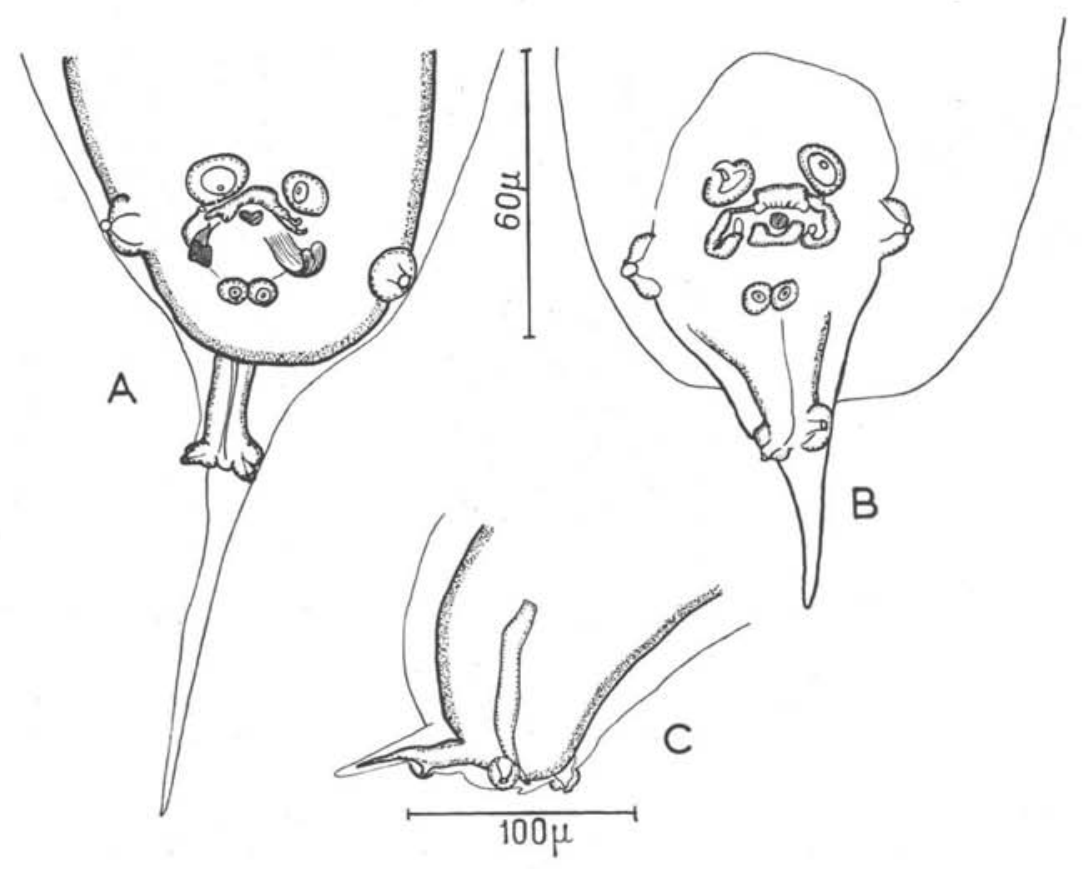

FIG. 15. - Thelandros meridionalis. Extrémité postérieure du mâle $\mathrm{A}$ et $\mathrm{C})$ Vues ventrale et latérale; spécimen de Zampongotra B) Vue ventrale; spécimen type

Bouche avec six petites lèvres, bien saillantes en avant et donnant à la tête un aspect crénelé lorsqu'elle est examinée en vue latérale. En vue apicale, la région buccale semble séparée du corps par un petit anneau chitinoïde de $40 \mu$ de diamètre. Les quatre lèvres submédianes portent chacune une très grosse papille et les deux lèvres latérales, une amphide de forme allongée. La cavité buccale est très faible et la lumière triradiée de l'œsophage apparaît juste en arrière du niveau de la bouche.

Une femelle longue de $6,8 \mathrm{~mm}$. est large de $950 \mu$. L'œsophage total est long de $1,39 \mathrm{~mm}$. et le bulbe lui-même de $210 \mu$. L'anneau nerveux et le pore excréteur sont respectivement à $110 \mu$ et $2,2 \mathrm{~mm}$. de l'extrémité antérieure. Queue longue de $285 \mu$ avec pointe caudale aiguë de $85 \mu$. Vulve à $3,8 \mathrm{~mm}$. de l'extrémité antérieure.

Ovéjecteur complexe différencié en plusieurs segments figurés en 16 A. Deux utérus. Ovaires enroulés autour de la partie postérieure de l'œsophage. Eufs asymétriques, à surface finement ponctuée, mesurant $115 \mu \times 62 \mu$. 

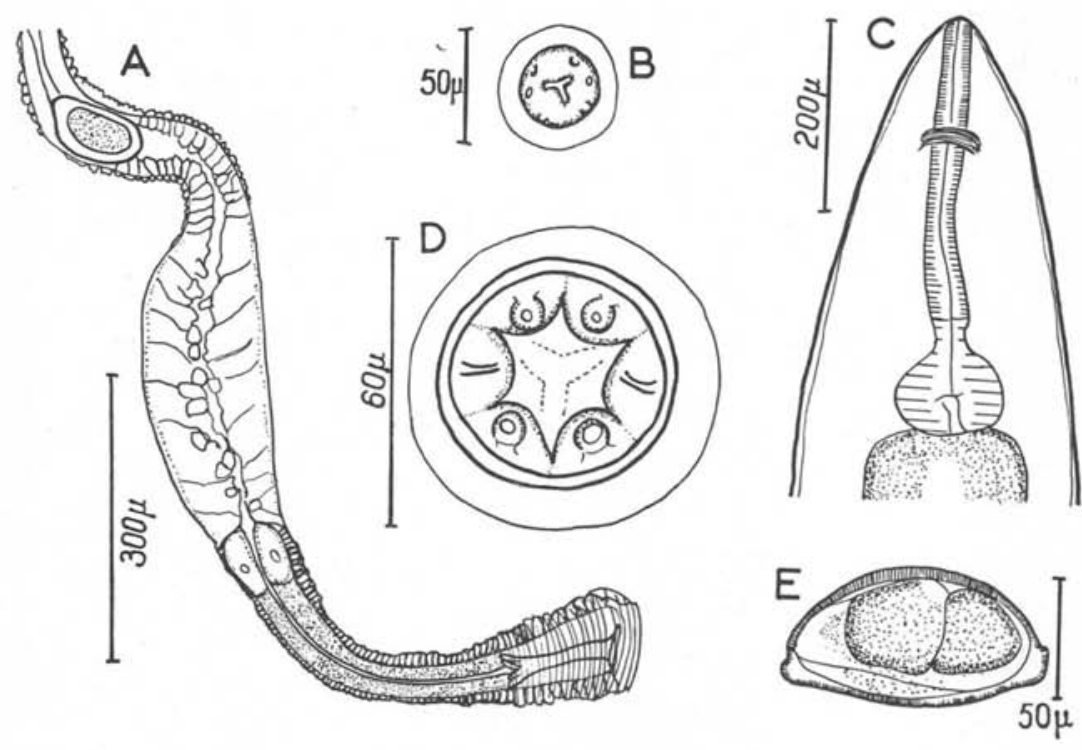

FIG. 16. - Thelandros meridionalis. A) Ovéjecteur. - B) Tête, mâle, vue apicale. C) Extrémité antérieure, mâle. - D) Tête, femelle, vue apicale. - E) CEuf.

\section{Discussion}

Les espèces décrites dans le genre Thelandros Wedl 1862 [Parapharyngodon Chatterji (1933)] sont nombreuses, mais les deux papilles de la paire postcloacale sont très rarement coalescentes. En outre, l'ornementation chitinoïde péricloacale, formée d'un cercle se soulevant en petites languettes latérales, semble caractéristique. Nous pensons donc que l'espèce est nouvelle et proposons le nom de Thelandros meridionalis $\mathrm{n}$. sp.

\section{Strongyluris RADAODYI Chabaud et Brygoo 1960}

L'espèce est fréquente chez de nombreux Caméléons de régions variées. Nous la signalons à nouveau dans cette note, d'une part parce que l'on peut trouver des spécimens d'une taille très supérieure à ce que nous avions observé (longueur du mâle : $9 \mathrm{~mm}$., longueur de la femelle : $13 \mathrm{~mm}$., chez un Chamaeleo brevicornis de Périnet), et, d'autre part, parce que nous l'avons identifiée chez un Lézard, Zonosaurus maximus Boulenger, provenant du Nord d'Amboasary (24 janvier 1962).

\section{FOLEYELLA BREVICAUDA n.Sp.}

Un Chamaeleo brevicornis, capturé à Périnet en février 1962, a révélé au cours d'un examen de routine une Filaire femelle, sous la peau de la queue. 

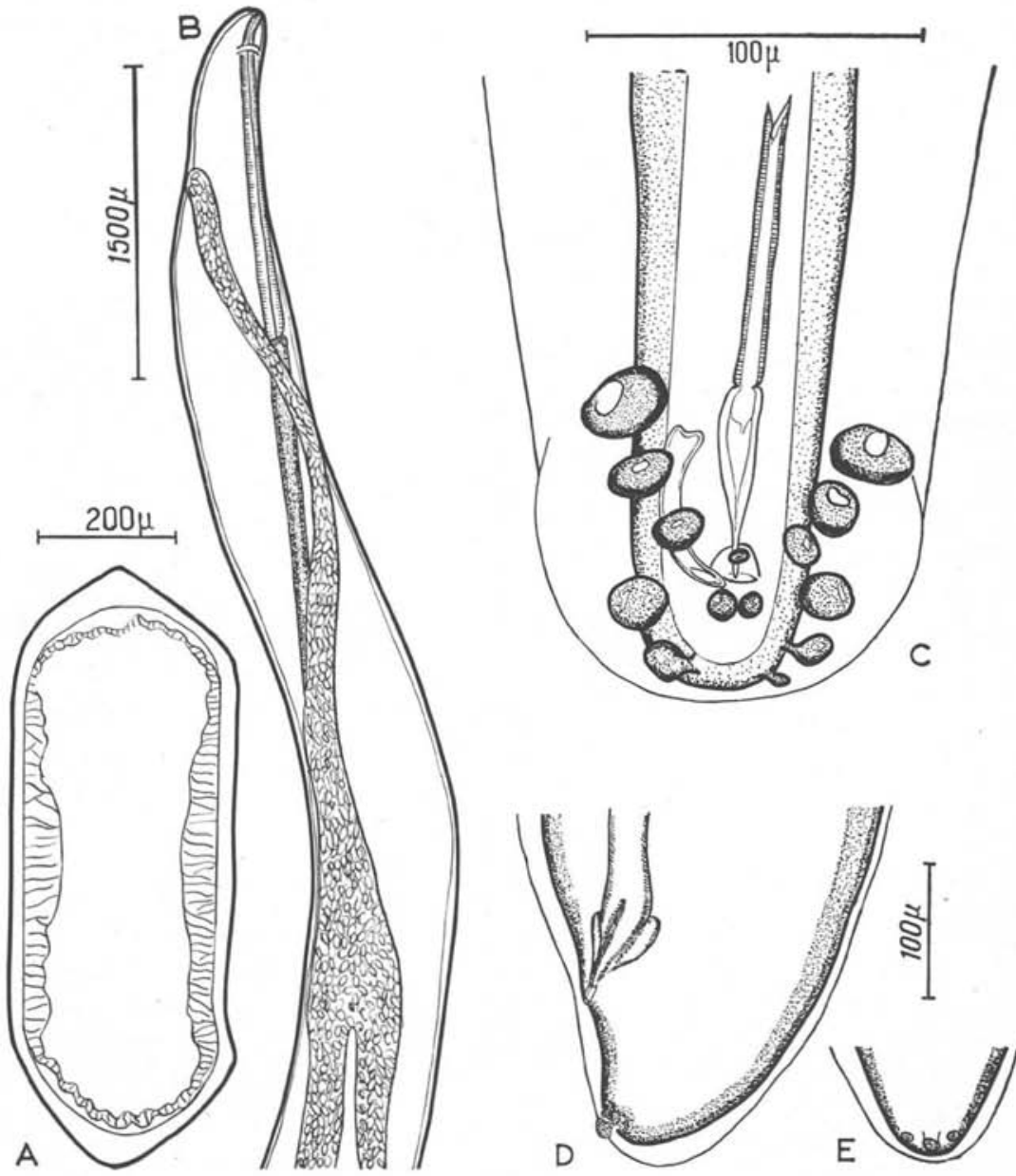

FIG. 17. - Foleyella brevicauda. A) Coupe du corps (axe latéral vertical). B) Extrémité antérieure, femelle, vue latérale. - C) Extrémité postérieure, mâle, vue ventrale. - D) Extrémité postérieure, femelle, vue latérale. - E) Pointe caudale, femelle, vue ventrale.

Cette Filaire a pu être extraite sur le vivant, puis lorsque, ultérieurement, l'animal a été sacrifié, 4 mâles et 7 femelles ont été trouvés dans les muscles superficiels des cuisses (matériel type). Un autre $C$. brevicornis, capturé à Ambavaniasy le 26 janvier 1962, portait également, dans les muscles de la cuisse, un mâle et une femelle d'une Filaire appartenant à la même espèce. Les deux Caméléons avaient dans le sang des microfilaires différentes de 
celles de Foleyella furcata. En outre, un Chamaeleo parsonii d'Ambavaniasy (20 décembre 1962), chez lequel n'avait été trouvé qu'un spécimen de Foleyella furcata, présentait néanmoins dans le sang des microfilaires identiques à celles de $F$. brevicauda.

\section{Description}

Corps cylindrique, avec extrémité postérieure très atténuée. L'intestin contient une substance jaune vif, qui apparaît nettement à l'œil nu.

La cuticule est assez épaisse et porte des stries transversales fines et rapprochées. Elle est très épaissie sur les axes latéraux et forme deux ailes latérales obtuses. La paroi musculaire est très fine sur les axes latéraux et devient épaisse sur les axes médians.

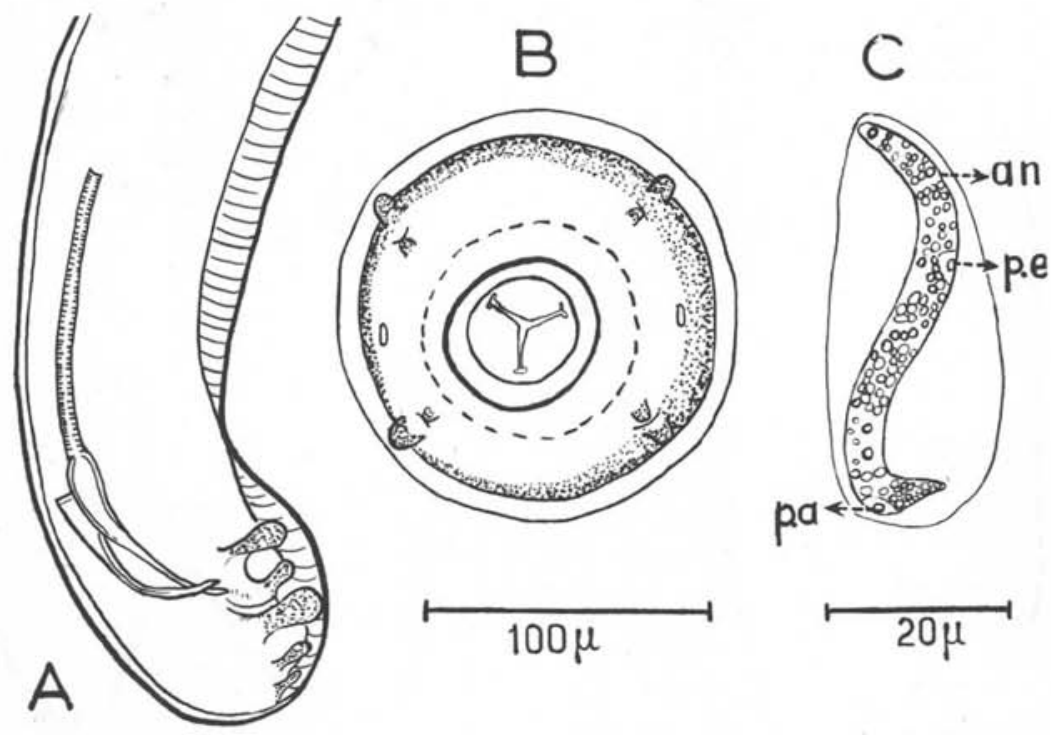

FIG. 18. - Foleyella brevicauda. A) Extrémité postérieure, mâle, vue latérale. B) Tête, femelle, vue apicale. - C) Microfilaire.

La bouche, limitée par un anneau faiblement chitinoïde, est circulaire. Elle est grande (diamètre interne : $15 \mu$ ) ; il n'y a pas de capsule buccale, les trois lobes œsophagiens atteignant presque le niveau buccal.

Les papilles sont réparties sur deux cycles, un cycle interne avec deux amphides et quatre papilles submédianes assez petites, et un cycle externe avec quatre papilles submédianes plus grandes.

Esophage relativement long, avec séparation musculo-glandulaire perceptible. Anneau nerveux à la partie antérieure de l'œsophage glandulaire; pore excréteur un peu en arrière; diérides non visibles. 
Femelle : Corps long de 62 à $70 \mathrm{~mm}$. Chez une femelle de $65 \mathrm{~mm}$., la largeur maximum est de $800 \mu$.

L'œsophage musculaire et l'œsophage glandulaire sont longs respectivement de $200 \mu$ et $1.450 \mu$. L'anneau nerveux et le pore excréteur sont situés respectivement à 240 et $330 \mu$ de l'apex. La vulve, non saillante, s'ouvre à $900 \mu$ de lextrémité antérieure.

L'ovéjecteur se dirige directement vers l'arrière sur une longueur de $4,4 \mathrm{~mm}$. ; il se dilate progressivement en arrière et se divise en deux utérus. Il peut y avoir sur certains spécimens une courbe récurrente de l'ovéjecteur avant sa division. Les parois musculaires de l'ovéjecteur restent épaisses sur une très longue distance, et il n'y a pas de région sphinctérienne particulièrement différenciée. La limite cuticulaire du vagin ne peut être précisée.

Les deux utérus contiennent peu de microfilaires. Ils descendent parallèlement l'un à l'autre jusqu'à l'extrémité postérieure, en ne décrivant que très peu de sinuosités. Les ovaires et oviductes sont repliés dans les derniers millimètres du corps.

Le diamètre du corps se rétrécit fortement à environ $1 \mathrm{~mm}$. de la pointe caudale, et la cuticule forme sur cette partie terminale du corps de véritables ailes caudales. La queue proprement dite est arrondie et très courte $(120 \mu)$; elle est faiblement courbée ventralement et se termine par un léger bouton arrondi, entouré de deux grosses phasmides subterminales. L'anus est peu perceptible et les parois rectales très faibles.

Mâle : Sur un spécimen long de $15,4 \mathrm{~mm}$., la largeur maximum est de $250 \mu$. L'œsophage est long de $1.250 \mu$ (partie musculaire : $170 \mu$, partie glandulaire : $1.080 \mu$ ). L'anneau nerveux est à $180 \mu$ de l'apex.

L'extrémité postérieure est fortement amoindrie sur une longueur de $1,7 \mathrm{~mm}$., mais, à ce niveau, les ailes caudales sont présentes et si développées que le diamètre du corps sur l'axe latéral est à peine diminué.

La queue est très courte $(35 \mu)$ et les papilles cloacales peu nombreuses. Il y a habituellement trois paires et une paire précloacales, deux paires alignées en arrière du cloaque et une ou deux paires subterminales.

Le spicule droit, long de $70 \mu$, est fortement concave ventralement. Le gauche, long de $185 \mu$, comprend un manche cylindrique, long de $95 \mu$, et une lame dilatée à la base et se terminant en pointe très aiguë.

Microfilaire : Des frottis de sang ont été pratiqués sur le Caméléon, chez lequel a été prélevé le matériel type, et ont été colorés au Giemsa.

Les microfilaires ne sont pas incluses dans une gaine, mais dans une coque souple et dépressible, de forme ovalaire (environ $42 \mu \times 18 \mu$ ), comparable à celle qui a été décrite récemment pour une autre filaire de Reptile, Macdonal. dius oschei. Le corps est court et trapu. Les noyaux sont petits et serrés. Espace céphalique très court, avec un noyau isolé. Queue brusquement rétrécie, assez arrondie, peu différente de l'extrémité céphalique. Les dimensions 
principales (moyennes sur 10 spécimens) sont: longueur: 62,7 $\mu$, largeur : $4,5 \mu$. Anneau nerveux et pore excréteur respectivement à $10 \mu$ et à $20 \mu$ de l'apex. Il existe une zone claire, dont les limites antérieures et postérieures sont à peu près à 35 et $45 \mu$ de l'apex, dans laquelle se trouvent deux ou trois grands noyaux isolés qui correspondent peut-être à des cellules rectales. Le pore anal est à $8 \mu$ de la pointe caudale.

\section{Discussion}

L'espèce reste très proche des autres Foleyella, parasites de Caméléons ou d'Agames en Afrique et à Madagascar, c'est-à-dire de F candezei (Fraipont 1882 ) et de $F$. furcata (Linstow 1895). Cependant, elle s'en distingue aisément : a) par la longueur de l'œsophage (femelle : 1,65 mm., mâle : 1,25 mm.), au lieu de 5 à $800 \mu$ chez les deux autres espèces ; $b$ ) par les ailes latérales, présentes comme chez candezei, mais d'aspect bien différent ; c) par les papilles cloacales, très groupées autour du cloaque et donnant à la queue un aspect raccourci ; $d$ ) par la miorofilaire incluse dans une enveloppe ovalaire.

Les noms de $F$. chamaeleonis Kreis 1938 ou de $F$. pigmentata Kreis 1945 , que nous considérons comme synonymes de $F$. furcata (cf. Chabaud, Anderson et Brygoo, 1960), ne peuvent être retenus, car, bien que l'œsophage atteigne $1.008 \mu$ chez la femelle de $F$. pigmentata, les caractères ne coïncident pas avec ceux décrits plus haut.

\section{RESUME ET CONCLUSION}

Description de Oswaldocruzia legendrei n. sp., parasite de Chamaeleo brevicornis (hôte-type), de C. lateralis et de Brookesia superciliaris (espèce caractérisée par un œsophage rétréci en isthme dans sa partie moyenne, par la queue de la femelle portant deux petits tubercules en plus de la pointe terminale, et par l'anatomie des spicules dépourvus de pointes accessoires et ayant deux pointes ventrales symétriques en forme de crochets).

Description de Raillietnema travassosi n. sp., parasite de Chamaeleo nasutus (taille relativement grande ; environ cinq œufs dans les utérus, gubernaculum presque aussi long que les spicules).

Description de Raillietnema vicarians n. sp., parasite de Chamaeleo gallus (environ 12 œufs dans les utérus, gubernaculum et spicules très courts).

Description de Raillietnema deblocki n. sp., parasite de Chamaeleo parsonii (environ 24 œufs dans les utérus, gubernaculum court, spicules longs).

Description de Raillietnema dupuisi n. sp., parasite de Chamaeleo parsonii (environ 5 œufs dans les utérus, gubernaculum court, spicules longs).

Description de Raillietnema oligogenos n. sp., parasite de Chamaeleo minor ( 1 ou 2 œufs dans les utérus, gubernaculum petit, spicules de taille moyenne). 
Description de Pharyngodon dimorpha n. sp., parasite de Chamaeleo pardalis et de Hoplurus cyclurus (espèce proche de celles qui sont décrites d'Australie, mais pourvue de petites ailes céphaliques transparentes chez la larve et le mâle, et d'une pièce chitinoïde complexe supportant le cône génital).

Description de Thelandros meridionalis n. sp., parasite de Hoplurus sp. (hôte-type), Hoplurus cyclurus, Acrantophis dumerilii, Chamaeleo verrucosus et $C$. lateralis (espèce identifiable par la coalescence des deux papilles de la paire postcloacale et par l'ornementation chitinoïde péricloacale).

Description de Foleyella brevicauda n. sp., parasite de Chamaeleo brevicornis et de $C$. parsonii (espèce proche de Foleyella furcata mais distincte par la longueur de l'œsophage, l'aspect des ailes latérales et des papilles cloacales et par la microfilaire incluse dans une gaine ovalaire).

Le présent travail, basé sur 530 dissections de Caméléons appartenant à 17 espèces différentes et en provenance de 54 localités réparties dans toute l'Ile, porte donc à 20 le nombre des espèces découvertes chez les Caméléons malgaches.

Si nous cherchons à schématiser leur type de répartition, nous pouvons les diviser en plusieurs groupes:

- A : quatre espèces accidentelles.

a) Falcaustra golvani, parasite banal d'Amphibien trouvé une fois chez un Caméléon.

b) Pharyngodon dimorpha, parasite d'Hoplurus, trouvé une fois chez un Caméléon, alors que 21 autres Caméléons de la même espèce, de la même localité, examinés à la même époque, étaient négatifs.

c) Thelandros meridionalis, parasite d'Hoplurus, dont une ou deux femelles ont été trouvées chez un Serpent et à quatre reprises chez des Caméléons dans les cantons méridionaux de l'Ile.

d) Thelandros sp. Chabaud et Brygoo 1960, un seul spécimen mâle a été trouvé à Nossi-Bé. En dehors des Caméléons, une bonne collection d'Oxyures de Reptiles a été rassemblée mais n'est pas encore étudiée. Nous ne pouvons donc rien conclure en ce qui concerne cette espèce, mais sommes tentés à titre provisoire de supposer qu'il s'agit, comme pour les Oxyures précédents, d'un parasitisme accidentel chez les Caméléons.

- B : six espèces ayant à la fois une spécificité et une distribution géographique étroites.
a) Raillietnema travassosi.
b) $R$. vicarians.
c) R. deblocki.
d) $R$. dupuisi.
e) $R$. oligogenos.
f) Maxvachonia dimorpha. 
Chacune de ces six espèces n'a été trouvée que chez un hôte déterminé dans une localité donnée. La spécificité semble très étroite puisque dans la même localité d'Ambavaniasy trois Caméléons hébergent chacun une espèce particulière de Raillietnema $(R$. deblocki chez $C$. parsonii, $R$. travassosi chez $C$. nasutus, $R$. vicarians chez $C$. gallus), mais la localisation géographique est également étroite puisque le même $C$. parsonii est parasité par $R$. deblocki à Ambavaniasy et par $R$. dupuisi à Vavatenina.

Nous rangeons provisoirement Maxvachonia dimorpha dans cette catégorie, mais la possibilité d'un parasitisme accidentel chez un Caméléon ne pourra être exclue que lorsque la faune des Reptiles en général sera mieux connue.

- $\mathrm{C}$ : deux espèces ayant une spécificité large, mais une distribution géographique étroite.

a) Oswaldocruzia legendrei, parasite de Brookesia et de plusieurs espèces de Caméléons, n’a jamais été trouvé ailleurs que dans la région de la Mandraka et d'Ambavaniasy-Périnet.

b) Foleyella brevicauda semble assez rare. Elle n'a été récoltée que chez C. brevicornis (deux fois) et chez $C$. parsonii (une fois) dans la même région d'Ambavaniasy-Périnet.

- D : huit espèces ayant une spécificité parasitaire et une distribution géographique larges :

a) Foleyella furcata dans les tissus sous-cutanés.

b) Rhabdias gemellipara dans les poumons (semblant cependant moins fréquent que les sept autres espèces).

c) et d) Abbreviata madagascariensis et Physalopteroides chamaeleonis dans l'estomac.

e) Hexametra angusticaecoides dans l'intestin antérieur.

f), g) et h) Spinicauda inglisi, Sp. freitasi et Strongyluris radaodyi dans l'intestin postérieur.

Quelques remarques générales semblent pouvoir être faites :

A) Chacune des vingt espèces est particulière à l'Ile, mais en dehors de Maxvachonia, qui est peut-être accidentel chez les Caméléons, il n’y a pas de genre endémique et la composition générale de la faune en Nématodes ne diffère pas profondément de celle des Caméléons d'autres régions du monde. (L'observation inverse a été faite récemment (1961) en ce qui concerne la faune des Nématodes d'Insectivores malgaches, dont la composition n'a aucun rapport avec celle des Insectivores des autres régions du monde).

B) Nous avons plus haut schématisé les types de répartition en quatre groupes, mais n'avons aucun exemple d'une spécificité parasitaire étroite associée à une distribution géographique étendue. Les faunes relictes paraissent se perpétuer en des points géographiques déterminés et non chez certains hôtes particuliers. 
Les notions classiques de parasitologie (basées essentiellement sur l'étude de groupes anciens tels que Protozoaires et Cestodes) montrent que la spécificité parasitaire joue un rôle plus important que la répartition géographique. Au contraire, pour les Nématodes, qui constituent apparemment un groupe d'évolution plus récente, nous avons eu l'occasion de signaler à différentes reprises l'existence du phénomène inverse. Les Nématodes de Caméléons malgaches offrent un exemple particulièrement net du rôle prépondérant joué par la répartition géographique dans le phénomène de la spéciation.

C) Les types de répartition des différentes espèces semblent liés à leur place systématique et à la date de leur adaptation au parasitisme.

Les Oxyuroidea sont essentiellement des parasites de Reptiles herbivores et il n'est pas surprenant de les voir figurer dans le groupe des parasites accidentels.

Les six espèces ayant une distribution et une spécificité étroites sont des Cosmocercoidea, groupe peut-être très ancien, que l'on place à la base des Ascaridida.

Parmi les deux espèces géographiquement localisées, l'Oswaldocruzia appartient à un phylum vraisemblablement très ancien. Ce n'est pas le cas pour Foleyella brevicauda, mais l'espèce n'est peut-être qu'une forme locale de F. furcata.

Parmi les huit espèces ayant une distribution et une spécificité larges, sept appartiennent à des phylums apparemment plus récents (Ascaridoidea, Heterakoidea, Spirurida). Le Rhabdias n'est pas localisé à un seul hôte et une seule région, mais il est moins fréquent et moins répandu que les sept autres espèces; il semble tendre à une répartition discontinue.

Nous pourrions donc conclure, pour schématiser en quelques lignes les résultats de ce travail :

a) La faune des Nématodes, parasites de Caméléons malgaches, est endémique à l'échelle spécifique, mais non générique.

b) Chacun des principaux organes des Caméléons est parasité par un Nématode appartenant à un phylum relativement récent. Le Nématode se retrouve chez tous les Caméléons et dans toute l'étendue de l'Ile.

c) Il existe en outre d'assez nombreuses espèces appartenant à des phylums plus anciens; elles ont alors une distribution géographique très localisée.

\section{Bibliographie}

BAYLIS (H. A.), 1929. - Some parasitic Nematodes from the Uluguru and Usambara Mountains, Tanganyika Territory. Ann. Mag. Nat. Hist., Ser. 10, IV, 372-381, fig. 1-3.

BaYlis (H. A.), 1930. - Some Heterakidae and Oxyuridae (Nematoda) from Queensland. Ann. Mag. Nat. Hist., Ser. 10, V, 354-366, fig. 1-10.

Ballesteros Marquez (A.), 1945. - Revision de la familia Cosmocercidae, Travassos, 1925. Rev. Iber. Parasit., Tome extraordinario, 150-180. 
Снabaud (A.-G.), 1959. - Phénomène d'évolution régressive des structures céphaliques et classification des Nématodes Spiruroidea. Parassitologia, I, 11-20.

Chabaud (A.-G.), Anderson (R. C.) et Brygoo (E. R.), 1960. - Cinq Filaires de Reptiles malgaches. Mém. Inst. Sc. Madagascar, Sér. A, XIII, 103-126, fig. 1-11.

Chabaud (A.-G.) et Brygoo (E. R.), 1957. - Deux Nématodes parasites de Grenouilles à Madagascar : Falcaustra golvani n. sp. et Harantinema ambocaeca n. gen., n. sp. Ann. Parasit., XXXII, 385-397, fig. 1-6.

Chabaud (A.-G.) et Brygoo (E. R.), 1960. - Nématodes parasites de Caméléons malgaches. Mém. Inst. Sc. Madagascar, Sér. A, XIV, 125-159, fig. 1-15.

Chabaud (A.-G.), Brygoo (E. R.) et Petter (A. J.), 1961. - Description et caractères biologiques de deux nouveaux Rhabdias malgaches. Ann. Parasit., XXXVI, 752-763, fig. 1-5.

Chabaud (A.-G.) et Golvan (Y.-J.), 1957. - Miscellanea helminthologica maroccana XXIV. Nématodes parasites de Lézards de la Forêt de Nefifik. Arc. Inst. Past. Maroc, V, 447-469, fig. 1-6.

Chabaud (A.-G.), Petter (A.) et Brygoo (E. R.), 1961. - Trois Nématodes parasites de Hérissons malgaches. Bull. Soc. Zool. France, LXXXVI, 38-51, fig. 1-5.

Fitzsimmons (W. W.), 1961. - Raillietnema chamaeleo n. sp. from Chamaeleo melleri (Gray) the Nyassaland giant horned Chamelion. Parassitologia, III, 145-149, fig. 1-4.

Fotedar (D. N.), 1960. - On a new species of Oxysomatium Railliet and Henry, 1913 and some notes on the genera Oxysomatium and Aplectana. Jl. Helminth., XXXIV, 141-150, fig. 1-4.

Gendre (E.), 1911. - Sur une espèce nouvelle d'Oxysoma. Proc. Verb. Soc. Linn. Bordeaux, LXV, 95-97, fig. 1-6.

Gupta (S. P.), 1959. - Nematodes parasites of Vertebrates of East Pakistan. I Oxyuridae from Lizards (Gekko and Hemidactylus). Canad. J. Zool., XXXVII, 469-475, fig. 1-15.

Johnston (T. H.) and Mawson (P. M.), 1941. - Some Nematodes from Kangaroo Island, South Australia. Rec. South. Austral. Mus., VII, 146-148, fig. 1-14.

KREIS (H. A.), 1938. - Beiträge zur Kenntnis parasitischer Nematoden. VIII. Zentralb. f. Bakt. u. Parasit., Orig., CXLII, 329-352, fig. 1-12.

Mrranda (C.), 1924. - Alguns Nematodeos do genero Aplectana Railliet et Henry, 1916. Mem. Ins. Osw. Cruz., XVII, 45-48, Pl. II-IV.

SANDGROUND (J. H.), 1928. - Some new Cestodes and Nematodes parasites from Tanganyika Territory. Proc. Boston. Soc. Nat. Hist., XXXIX, 131-150.

Travassos (L.), 1925. - Contribuiçoes para o conhecimento da Fauna helmintologico dos Batraquios do Brasil. Sciencia medica, III, 672-684, fig. 1-10.

Travassos (L.), 1927. - Schrankia brasili. Oxyuroidea de Batrachio. Bol. Biol., IX, 147-152, fig. 1-4.

Walton (A. C.), 1940. - The Nematode genus Raillietnema Travassos 1927. J. Wash. Acad. Sc., XXX, 474-478, fig. $1 a-3 b$.

Muséum National d'Histoire Naturelle (Zoologie des Vers), Paris et Institut Pasteur de Madagascar, Tananarive 